Supplement of Atmos. Chem. Phys., 15, 1421-1434, 2015

http://www.atmos-chem-phys.net/15/1421/2015/

doi:10.5194/acp-15-1421-2015-supplement

(C) Author(s) 2015. CC Attribution 3.0 License.

(c) (i)

Supplement of

Scavenging ratios of polycyclic aromatic compounds in rain and snow in the Athabasca oil sands region

\section{Zhang et al.}

Correspondence to: L. Zhang (leiming.zhang@ec.gc.ca)

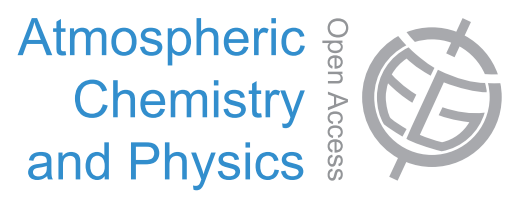

Atmospheric and Physics 


\section{Supplementary Materials}

Table S1: Monthly precipitation concentrations (ng/L). IDL = instrument detection limit (Pages 2 to 10)

Table S2: Monthly average air concentrations $\left(\mathrm{ng} / \mathrm{m}^{3}\right)$ based on 4-5 weekly air concentrations. NA = weekly air samples with recovery standard percentage below $50 \%$ or above $150 \%$. Dash - indicates no samples were collected (Pages 11 to 19$)$

Table S3: Descriptive statistics of the total scavenging ratios $\left(\mathrm{W}_{t}\right)$ for snow and rain at the oil sands sites. $(\mathrm{G})$ and (P) denote predominantly gas-phase and particulate-phase PACs, respectively (Page 20)

\begin{tabular}{|l|l|l|l|}
\hline Abbreviation & Full name & Abbreviation & Full name \\
\hline NAP & Naphthalene & BbFLT & Benzo $(b)$ fluoranthene \\
\hline AL & Acenaphthylene & BkFLT & Benzo $(k)$ fluoranthene \\
\hline AE & Acenaphthene & BaPY & Benzo $(a)$ pyrene \\
\hline FL & Fluorene & PER & Perylene \\
\hline PHE & Phenanthrene & IP & Indeno $(l, 2,3$-cd $)$ pyrene \\
\hline AN & Anthracene & DahAN & Dibenz $(a, h)$ anthracene \\
\hline FLT & Fluoranthene & BghiPER & Benzo $(g h i)$ perylene \\
\hline PY & Pyrene & PHE/AN & Phenanthrene/Anthracene \\
\hline RET & Retene & FLT/PYR & Fluoranthene/Pyrene \\
\hline BaAN & Benz $(a)$ anthracene & BTC & Benz $(a)$ anthracene/Triphenylene/Chrysene \\
\hline CHR & Chrysene & DBT & Dibenzothiophene \\
\hline
\end{tabular}


Table S1: Monthly precipitation concentrations (ng/L)

\begin{tabular}{|c|c|c|c|c|c|c|c|c|c|c|c|c|c|c|c|c|c|}
\hline Site & Start date & NAP & $\mathrm{AL}$ & $\mathrm{AE}$ & $\mathrm{FL}$ & PHE & AN & FLT & PY & RET & $\mathrm{BaAN}$ & $\mathrm{CHR}$ & BbFLT & BkFLT & BaPY & PER & IP \\
\hline AMS5 & $02 / 01 / 2011$ & IDL & 0.1 & 3.1 & 13.4 & 109.6 & 33.0 & 43.9 & 128.5 & IDL & 251.4 & 370.8 & 136.3 & 28.1 & 194.0 & 20.0 & 61.8 \\
\hline AMS5 & $31 / 01 / 2011$ & 841.8 & 3.9 & 10.3 & 52.2 & 567.8 & 209.7 & 169.9 & 567.1 & IDL & 786.2 & 1109.6 & 344.0 & 92.3 & 668.3 & 57.3 & 137.1 \\
\hline AMS5 & $02 / 03 / 2011$ & 4862.7 & 9.9 & 44.7 & 148.5 & 1404.0 & 796.6 & 556.0 & 1631.9 & 176.9 & 1902.9 & 3109.1 & 1708.3 & 250.0 & 3948.9 & 182.5 & 352.3 \\
\hline AMS5 & $01 / 04 / 2011$ & 2166.6 & 4.4 & 16.9 & 80.7 & 773.9 & 337.8 & 195.3 & 764.6 & 43.5 & 871.1 & 1333.9 & 308.0 & 111.1 & 979.0 & 75.8 & 167.0 \\
\hline AMS5 & $01 / 05 / 2011$ & 3876.0 & 8.8 & 54.6 & 33.9 & 227.8 & 85.1 & 50.0 & 195.0 & 25.9 & 203.7 & 376.0 & 72.7 & 21.0 & 208.5 & 21.4 & 35.5 \\
\hline AMS5 & $02 / 06 / 2011$ & 243.3 & $1.9 \mathrm{E}-02$ & IDL & 3.1 & 18.0 & 3.1 & 4.2 & 12.4 & 0.2 & 10.0 & 17.4 & 4.2 & 1.0 & 7.1 & 0.9 & 1.0 \\
\hline AMS5 & $19 / 07 / 2011$ & IDL & $3.1 \mathrm{E}-03$ & IDL & 1.3 & 9.9 & 1.5 & 4.0 & 6.0 & 50.1 & 5.2 & 8.7 & 2.5 & 0.2 & 3.7 & 1.0 & 0.8 \\
\hline AMS5 & $02 / 08 / 2011$ & 534.8 & 1.7E-02 & IDL & 1.6 & 10.4 & 1.9 & 2.9 & 9.8 & 7.7 & 7.8 & 12.9 & 2.9 & 0.9 & 5.5 & 0.8 & 0.9 \\
\hline AMS5 & $01 / 09 / 2011$ & 43.5 & 1.2 & 2.6 & 2.5 & 15.8 & 2.2 & 4.8 & 10.3 & 10.1 & 6.3 & 11.4 & 3.8 & 5.9E-02 & 6.1 & 2.5 & 1.7 \\
\hline AMS5 & $30 / 09 / 2011$ & 138.8 & $3.4 \mathrm{E}-02$ & 4.9 & 4.4 & 20.4 & 4.6 & 6.1 & 17.2 & 10.3 & 14.7 & 22.7 & 5.8 & IDL & 12.1 & 1.2 & 1.3 \\
\hline AMS5 & $01 / 11 / 2011$ & 0.5 & 1.2 & 2.2 & 2.5 & 24.3 & 3.2 & 9.6 & 18.8 & 29.4 & 10.2 & 20.1 & 8.8 & 0.4 & 8.9 & 5.0 & 3.6 \\
\hline AMS5 & $30 / 11 / 2011$ & 90.9 & 0.1 & 1.3 & 8.2 & 31.6 & 3.0 & 16.4 & 27.9 & 12.0 & 12.3 & 17.0 & 7.6 & 2.0 & 2.0 & 1.8 & 2.3 \\
\hline AMS5 & $30 / 12 / 2011$ & 41.6 & 1.4 & 2.8 & 13.0 & 134.1 & 43.9 & 46.3 & 144.6 & 88.3 & 200.5 & 255.8 & 71.4 & 20.9 & 188.3 & 14.2 & 33.0 \\
\hline AMS5 & $31 / 01 / 2012$ & 95.3 & 2.3 & 6.7 & 38.3 & 356.1 & 134.6 & 104.9 & 339.3 & 167.7 & 355.8 & 469.9 & 160.8 & 30.4 & 397.8 & 33.8 & 78.7 \\
\hline AMS5 & $29 / 02 / 2012$ & 2497.5 & 40.0 & 118.8 & 521.3 & 5173.8 & 2092.5 & 1731.3 & 5780.0 & 1201.3 & 7672.5 & $1.1 \mathrm{E}+04$ & 2772.5 & 612.5 & 6960.0 & 572.5 & 1242.5 \\
\hline AMS5 & $30 / 03 / 2012$ & 23.2 & 0.4 & 1.5 & 9.0 & 83.4 & 26.2 & 25.1 & 81.1 & 9.0 & 103.9 & 141.8 & 38.3 & 9.8 & 77.3 & 7.0 & 19.1 \\
\hline AMS5 & $30 / 04 / 2012$ & 0.6 & 0.4 & 0.8 & 3.5 & 32.1 & 7.2 & 14.1 & 29.1 & 8.3 & 23.5 & 35.8 & 12.3 & 2.6 & 22.0 & 4.8 & 6.3 \\
\hline AMS5 & $31 / 05 / 2012$ & 26.0 & 0.7 & 2.9 & 4.2 & 40.2 & 7.3 & 12.2 & 31.0 & 33.1 & 33.2 & 43.3 & 22.0 & 3.5 & 27.3 & 8.3 & 9.0 \\
\hline
\end{tabular}


Table S1: Monthly precipitation concentrations (ng/L) cont'd

\begin{tabular}{|c|c|c|c|c|c|c|c|c|c|c|c|c|c|c|c|c|c|}
\hline Site & Start date & NAP & $\mathrm{AL}$ & $\mathrm{AE}$ & $\mathrm{FL}$ & PHE & AN & FLT & PY & RET & $\mathrm{BaAN}$ & $\mathrm{CHR}$ & $\mathrm{BbFLT}$ & BkFLT & $\mathrm{BaPY}$ & PER & IP \\
\hline AMS11 & 02/01/2011 & 2558.7 & 4.3 & 7.7 & 36.4 & 350.4 & 115.2 & 133.5 & 369.7 & 172.4 & 503.1 & 695.7 & 234.5 & 51.6 & 370.8 & 36.4 & 98.9 \\
\hline AMS11 & $31 / 01 / 2011$ & 474.9 & 3.2 & 11.7 & 82.6 & 831.8 & 314.8 & 271.8 & 901.0 & IDL & 1440.3 & 1934.9 & 606.7 & 161.1 & 1263.6 & 113.2 & 257.3 \\
\hline AMS11 & $02 / 03 / 2011$ & 5836.3 & 12.7 & 70.9 & 255.9 & 2268.9 & 1133.1 & 591.9 & 2191.6 & 159.8 & 2377.1 & 3333.6 & 545.5 & 199.3 & 4650.8 & 208.5 & 404.3 \\
\hline AMS11 & $01 / 04 / 2011$ & 4499.5 & 4.0 & 20.3 & 99.6 & 838.1 & 365.2 & 203.5 & 778.4 & 43.6 & 951.8 & 1439.4 & 336.9 & 95.2 & 1049.1 & 93.5 & 173.0 \\
\hline AMS11 & $30 / 04 / 2011$ & $1.0 \mathrm{E}+04$ & 4.7 & 33.4 & 168.0 & 1494.1 & 726.2 & 367.4 & 1547.8 & 46.5 & 1874.2 & 2856.9 & 659.5 & 137.8 & 1815.5 & 169.8 & 295.4 \\
\hline AMS11 & 02/06/2011 & 555.7 & $2.5 \mathrm{E}-02$ & 3.6 & 12.4 & 110.7 & 38.2 & 29.2 & 114.4 & 81.5 & 138.0 & 195.2 & 50.3 & 12.9 & 116.0 & 10.4 & 20.2 \\
\hline AMS11 & $19 / 07 / 2011$ & 13.8 & $3.8 \mathrm{E}-03$ & IDL & 3.0 & 26.5 & 9.2 & 7.4 & 25.0 & 58.3 & 33.0 & 46.4 & 12.1 & 2.2 & 27.8 & 2.7 & 5.2 \\
\hline AMS11 & $02 / 08 / 2011$ & 138.7 & $1.0 \mathrm{E}-02$ & IDL & 2.6 & 25.7 & 7.2 & 6.7 & 20.6 & 0.1 & 27.3 & 39.6 & 11.5 & 0.5 & 21.8 & 3.0 & 4.1 \\
\hline AMS11 & 01/09/2011 & 353.1 & 4.7E-02 & 16.0 & 21.8 & 128.8 & 44.1 & 38.2 & 121.6 & IDL & 132.0 & 184.9 & 49.9 & 13.8 & 123.2 & 17.5 & 22.9 \\
\hline AMS11 & $30 / 09 / 2011$ & 45.8 & 0.4 & 3.8 & 6.2 & 39.7 & 14.8 & 10.1 & 36.2 & 12.0 & 43.6 & 60.6 & 15.4 & 3.6 & 38.8 & 5.0 & 6.9 \\
\hline AMS11 & $01 / 11 / 2011$ & 5.2 & 0.2 & 2.0 & 3.5 & 40.2 & 10.3 & 13.6 & 40.7 & 31.0 & 49.6 & 64.5 & 19.4 & 3.4 & 40.5 & 6.2 & 5.9 \\
\hline AMS11 & $30 / 11 / 2011$ & 2675.0 & IDL & 55.0 & 870.0 & $1.1 \mathrm{E}+04$ & 4087.5 & 3317.5 & $1.3 \mathrm{E}+04$ & 30.0 & $1.9 \mathrm{E}+04$ & $2.2 \mathrm{E}+04$ & 5907.5 & 1280.0 & $1.6 \mathrm{E}+04$ & 1187.5 & 2462.5 \\
\hline AMS11 & $20 / 12 / 2011$ & 92.3 & 2.5 & 8.7 & 36.0 & 335.5 & 123.7 & 96.2 & 357.4 & 31.7 & 520.9 & 635.7 & 182.1 & 44.7 & 486.5 & 34.6 & 83.0 \\
\hline AMS11 & $30 / 01 / 2012$ & 242.1 & 3.9 & 17.6 & 89.2 & 848.8 & 334.8 & 228.0 & 794.9 & 197.6 & 1087.9 & 1402.8 & 395.9 & 91.7 & 995.1 & 74.1 & 196.3 \\
\hline AMS11 & $29 / 02 / 2012$ & $1.2 \mathrm{E}+04$ & 106.7 & 823.3 & 4643.3 & $4.2 \mathrm{E}+04$ & $1.6 \mathrm{E}+04$ & $1.2 \mathrm{E}+04$ & $4.0 \mathrm{E}+04$ & 6090.0 & $5.6 \mathrm{E}+04$ & $7.1 \mathrm{E}+04$ & $2.0 \mathrm{E}+04$ & 5560.0 & $5.0 \mathrm{E}+04$ & 3893.3 & 9353.3 \\
\hline AMS11 & $30 / 03 / 2012$ & 44.6 & 0.5 & 3.1 & 17.6 & 172.9 & 69.6 & 48.4 & 181.2 & 9.0 & 254.4 & 322.6 & 90.0 & 20.7 & 231.5 & 17.7 & 46.4 \\
\hline AMS11 & $30 / 04 / 2012$ & 9.6 & 0.2 & 1.7 & 6.0 & 65.4 & 19.7 & 18.8 & 60.2 & 9.2 & 77.3 & 104.0 & 32.0 & 8.2 & 64.6 & 8.8 & 14.3 \\
\hline AMS11 & $31 / 05 / 2012$ & 23.0 & 0.5 & 3.5 & 3.9 & 26.1 & 2.8 & 5.6 & 14.5 & 23.9 & 17.0 & 23.0 & 9.8 & 1.6 & 12.9 & 3.6 & 4.3 \\
\hline
\end{tabular}


Table S1: Monthly precipitation concentrations (ng/L) cont'd

\begin{tabular}{|c|c|c|c|c|c|c|c|c|c|c|c|c|c|c|c|c|c|}
\hline Site & Start date & NAP & $\mathrm{AL}$ & $\mathrm{AE}$ & $\mathrm{FL}$ & PHE & AN & FLT & PY & RET & BaAN & $\mathrm{CHR}$ & $\mathrm{BbFLT}$ & BkFLT & BaPY & PER & IP \\
\hline AMS13 & $24 / 01 / 2011$ & 56.4 & 0.5 & 0.9 & 4.0 & 34.1 & 5.1 & 15.2 & 24.8 & 11.8 & 36.5 & 60.7 & 24.2 & 5.9 & 26.1 & 4.8 & 10.2 \\
\hline AMS13 & $31 / 01 / 2011$ & 1662.5 & 2.0 & 3.9 & 12.8 & 129.1 & 37.5 & 51.7 & 128.9 & 63.3 & 161.4 & 234.9 & 81.0 & 16.8 & 132.3 & 16.2 & 33.6 \\
\hline AMS13 & $02 / 03 / 2011$ & 2165.3 & 3.7 & 7.2 & 15.5 & 125.8 & 24.3 & 46.5 & 60.8 & 142.6 & 61.4 & 79.0 & 17.1 & 5.0 & 66.0 & 10.9 & 10.5 \\
\hline AMS13 & $01 / 04 / 2011$ & 1965.9 & 0.4 & 0.7 & 3.3 & 63.1 & 17.7 & 18.3 & 46.4 & 39.5 & 45.3 & 73.9 & 26.6 & 5.8 & 45.7 & 14.6 & 10.6 \\
\hline AMS13 & $30 / 04 / 2011$ & 3929.4 & 9.9 & 69.0 & 22.6 & 99.3 & 14.7 & 15.3 & 56.1 & 8.3 & 29.5 & 50.2 & 15.3 & 5.6 & 27.9 & 10.4 & 6.6 \\
\hline AMS13 & $02 / 06 / 2011$ & 11.4 & $3.8 \mathrm{E}-02$ & $\mathrm{IDL}$ & 2.7 & 24.2 & 4.3 & 6.6 & 21.7 & 0.4 & 18.3 & 30.8 & 7.6 & 1.9 & 13.1 & 1.7 & 1.9 \\
\hline AMS13 & $19 / 07 / 2011$ & 31.6 & $2.5 \mathrm{E}-03$ & $\mathrm{IDL}$ & 1.6 & 10.3 & 1.1 & 2.8 & 4.1 & 91.4 & 3.5 & 5.5 & 1.6 & 0.1 & 1.8 & 0.1 & 0.1 \\
\hline AMS13 & $02 / 08 / 2011$ & 121.7 & $1.0 \mathrm{E}-02$ & IDL & IDL & 2.8 & $5.0 \mathrm{E}-02$ & 0.6 & 0.9 & 1.8 & 0.3 & 0.2 & 0.3 & 0.5 & 0.5 & 0.5 & 0.5 \\
\hline AMS13 & 01/09/2011 & 28.1 & $1.2 \mathrm{E}-02$ & 1.4 & 1.3 & 5.7 & 0.4 & 1.2 & 2.1 & 3.5 & 1.9 & 2.4 & 0.8 & IDL & 0.6 & 0.5 & 0.6 \\
\hline AMS13 & $30 / 09 / 2011$ & 49.5 & 1.1 & 2.4 & 2.0 & 12.6 & 1.3 & 4.1 & 8.7 & 6.8 & 4.8 & 7.5 & 1.7 & IDL & 3.1 & 1.1 & 1.2 \\
\hline AMS13 & $01 / 11 / 2011$ & 13.3 & 0.2 & 0.9 & 2.5 & 28.2 & 6.5 & 10.8 & 26.1 & 26.7 & 27.4 & 37.3 & 11.3 & 0.4 & 23.8 & 2.2 & 3.8 \\
\hline AMS13 & $30 / 11 / 2011$ & 19.0 & IDL & 3.2 & 5.9 & 72.8 & 20.2 & 28.3 & 80.8 & 53.7 & 93.3 & 123.4 & 39.4 & 7.3 & 81.8 & 12.6 & 13.7 \\
\hline AMS13 & $30 / 12 / 2011$ & 29.9 & 1.4 & 1.3 & 6.1 & 54.5 & 11.8 & 24.6 & 50.3 & 57.4 & 53.1 & 70.3 & 22.9 & 3.7 & 48.2 & 5.9 & 13.5 \\
\hline AMS13 & $31 / 01 / 2012$ & 9.2 & 0.9 & 0.4 & 5.2 & 57.7 & 11.6 & 19.1 & 38.7 & 68.7 & 45.1 & 61.8 & 15.5 & 3.9 & 37.4 & 4.6 & 8.0 \\
\hline AMS13 & $29 / 02 / 2012$ & 109.7 & 0.7 & 6.5 & 43.5 & 523.2 & 150.0 & 161.2 & 469.3 & 235.2 & 615.0 & 833.2 & 207.7 & 41.7 & 547.0 & 55.7 & 103.7 \\
\hline AMS13 & $30 / 03 / 2012$ & 40.9 & 0.5 & 5.2 & 13.5 & 189.3 & 22.7 & 41.6 & 82.7 & 64.3 & 87.6 & 127.8 & 45.3 & 8.0 & 68.7 & 23.3 & 20.1 \\
\hline AMS13 & $30 / 04 / 2012$ & IDL & 0.1 & 1.1 & 1.4 & 22.8 & 1.9 & 5.4 & 11.3 & 15.5 & 9.5 & 14.6 & 6.3 & 1.2 & 6.2 & 3.5 & 2.8 \\
\hline AMS13 & $31 / 05 / 2012$ & 19.5 & 0.4 & 1.7 & 2.3 & 19.7 & 2.5 & 6.0 & 10.4 & 17.3 & 10.3 & 16.3 & 8.7 & 1.5 & 8.5 & 3.1 & 3.5 \\
\hline
\end{tabular}


Table S1: Monthly precipitation concentrations (ng/L) cont'd

\begin{tabular}{|c|c|c|c|c|c|c|c|c|c|c|c|c|c|c|c|c|c|}
\hline Site & Start date & DahAN & BghiPER & $\begin{array}{c}\text { C1 } \\
\text { NAP }\end{array}$ & $\begin{array}{c}\text { C2 } \\
\text { NAP }\end{array}$ & $\begin{array}{l}\text { C3 } \\
\text { NAP }\end{array}$ & $\begin{array}{c}\text { C4 } \\
\text { NAP }\end{array}$ & $\mathrm{C} 1 \mathrm{FL}$ & $\mathrm{C} 2 \mathrm{FL}$ & C3 FL & C4 FL & $\begin{array}{c}\text { C1 } \\
\text { PHE/AN }\end{array}$ & $\begin{array}{c}\mathrm{C} 2 \\
\text { PHE/AN }\end{array}$ & $\begin{array}{c}\text { C3 } \\
\text { PHE/AN }\end{array}$ & $\begin{array}{c}\text { C4 } \\
\text { PHE/AN }\end{array}$ & $\begin{array}{c}\text { C1 } \\
\text { FLT/PYR }\end{array}$ & $\begin{array}{c}\text { C2 } \\
\text { FLT/PYR }\end{array}$ \\
\hline AMS5 & $02 / 01 / 2011$ & 113.5 & 203.3 & 61.3 & 66.0 & 89.6 & 31.4 & 55.1 & 213.7 & 221.2 & 102.7 & 282.5 & 328.0 & 210.1 & 77.4 & 218.9 & 661.0 \\
\hline AMS5 & $31 / 01 / 2011$ & 255.7 & 458.3 & 346.6 & 406.9 & 446.3 & 143.9 & 244.5 & 663.5 & 1540.5 & 587.7 & 1719.8 & 2416.0 & 1879.3 & 1001.6 & 1106.9 & 2907.2 \\
\hline AMS5 & $02 / 03 / 2011$ & 879.2 & 1257.7 & 257.8 & 267.7 & 355.2 & 129.7 & 187.4 & 524.7 & 1273.1 & 519.8 & 1566.7 & 2499.6 & 1738.2 & 861.3 & 1107.1 & 2872.9 \\
\hline AMS5 & $01 / 04 / 2011$ & 278.6 & 564.5 & 442.9 & 462.6 & 432.3 & 172.0 & 219.1 & 537.1 & 1102.3 & 516.7 & 1657.5 & 2444.7 & 1713.1 & 926.3 & 1171.5 & 2973.2 \\
\hline AMS5 & $01 / 05 / 2011$ & 59.3 & 123.3 & 32.9 & 39.3 & 33.3 & 30.0 & 31.0 & 140.3 & 301.1 & 300.0 & 182.0 & 256.3 & 201.6 & 93.8 & 123.6 & 389.1 \\
\hline AMS5 & $02 / 06 / 2011$ & 0.6 & 5.5 & 12.7 & 15.6 & 22.5 & 26.8 & 5.5 & 43.7 & 70.0 & 70.6 & 43.4 & 74.5 & 83.5 & 39.6 & 24.6 & 81.7 \\
\hline AMS5 & $19 / 07 / 2011$ & 1.0 & 2.8 & 3.1 & 5.9 & 33.0 & 23.9 & 6.5 & 21.5 & 80.2 & 24.7 & 30.3 & 90.7 & 103.9 & 82.3 & 13.5 & 56.1 \\
\hline AMS5 & $02 / 08 / 2011$ & 0.5 & 4.1 & 7.0 & 3.7 & 14.1 & 8.9 & 6.1 & 15.3 & 42.5 & 32.1 & 24.8 & 56.9 & 63.6 & 48.4 & 16.1 & 61.3 \\
\hline AMS5 & 01/09/2011 & 1.8 & 5.0 & 7.5 & 10.8 & 19.0 & 15.4 & 6.6 & 19.3 & 42.7 & 36.2 & 35.4 & 92.8 & 107.7 & 75.9 & 18.0 & 72.5 \\
\hline AMS5 & $30 / 09 / 2011$ & 3.9 & 8.1 & 24.3 & 20.9 & 27.5 & 15.7 & 6.4 & 16.7 & 33.3 & 22.4 & 30.3 & 78.1 & 87.2 & 50.0 & 17.0 & 56.5 \\
\hline AMS5 & $01 / 11 / 2011$ & 2.3 & 8.7 & IDL & IDL & 24.9 & 34.0 & 11.4 & 41.0 & 145.6 & 109.8 & 76.3 & 217.2 & 299.4 & 211.9 & 41.6 & 186.6 \\
\hline AMS5 & $30 / 11 / 2011$ & 1.0 & 2.0 & 28.7 & 41.0 & 38.6 & 69.8 & 56.6 & 134.8 & 207.2 & 124.6 & 82.6 & 176.6 & 228.4 & 88.2 & 28.4 & 122.2 \\
\hline AMS5 & $30 / 12 / 2011$ & 67.2 & 108.9 & 70.6 & 95.1 & 95.6 & 54.9 & 58.1 & 146.5 & 321.7 & 164.8 & 401.5 & 604.0 & 435.0 & 245.2 & 262.6 & 721.3 \\
\hline AMS5 & $31 / 01 / 2012$ & 143.6 & 243.4 & 234.0 & 283.1 & 257.3 & 133.5 & 164.6 & 374.1 & 782.4 & 397.0 & 1060.3 & 1595.2 & 1098.9 & 687.5 & 651.2 & 1739.9 \\
\hline AMS5 & $29 / 02 / 2012$ & 2450.0 & 4232.5 & 4848.6 & 6114.3 & 5258.1 & 2431.4 & 2524.9 & 6450.5 & $1.3 \mathrm{E}+04$ & 5713.0 & $1.9 E+04$ & $3.0 \mathrm{E}+04$ & $2.0 \mathrm{E}+04$ & $1.1 \mathrm{E}+04$ & $1.4 \mathrm{E}+04$ & $3.6 \mathrm{E}+04$ \\
\hline AMS5 & $30 / 03 / 2012$ & 30.3 & 56.4 & 67.6 & 82.0 & 81.4 & 33.4 & 43.8 & 102.9 & 210.7 & 100.1 & 288.9 & 429.8 & 277.8 & 157.4 & 183.7 & 523.3 \\
\hline AMS5 & $30 / 04 / 2012$ & 7.7 & 17.5 & 7.4 & 14.6 & 39.7 & 29.0 & 15.6 & 51.5 & 127.2 & 85.5 & 112.5 & 246.5 & 235.4 & 140.1 & 59.3 & 196.9 \\
\hline AMS5 & $31 / 05 / 2012$ & 10.3 & 23.4 & 46.0 & 47.2 & 67.0 & 56.2 & 18.7 & 79.0 & 151.5 & 103.2 & 110.6 & 289.9 & 372.2 & 230.9 & 68.7 & 261.6 \\
\hline
\end{tabular}


Table S1: Monthly precipitation concentrations (ng/L) cont'd

\begin{tabular}{|c|c|c|c|c|c|c|c|c|c|c|c|c|c|c|c|c|c|}
\hline Site & Start date & DahAN & BghiPER & C1 NAP & C2 NAP & C3 NAP & C4 NAP & C1 FL & $\mathrm{C} 2 \mathrm{FL}$ & C3 FL & C4 FL & $\begin{array}{c}\text { C1 } \\
\text { PHE/AN }\end{array}$ & $\begin{array}{c}\mathrm{C} 2 \\
\text { PHE/AN }\end{array}$ & $\begin{array}{c}\text { C3 } \\
\text { PHE/AN }\end{array}$ & $\begin{array}{c}\text { C4 } \\
\text { PHE/AN }\end{array}$ & $\begin{array}{c}\text { C1 } \\
\text { FLT/PYR }\end{array}$ & $\begin{array}{c}\mathrm{C} 2 \\
\mathrm{FLT} / \mathrm{PYR}\end{array}$ \\
\hline AMS11 & 02/01/2011 & 151.7 & 298.7 & 247.6 & 258.9 & 208.6 & 93.3 & 152.2 & 365.7 & 994.0 & 407.6 & 1043.5 & 1592.5 & 1332.4 & 745.3 & 712.1 & 1885.5 \\
\hline AMS11 & $31 / 01 / 2011$ & 474.7 & 842.7 & 292.4 & 331.9 & 406.6 & 152.0 & 389.0 & 966.9 & 2191.1 & 1137.6 & 2450.5 & 3325.4 & 2656.4 & 1286.4 & 1695.4 & 4577.9 \\
\hline AMS11 & $02 / 03 / 2011$ & 826.4 & 1378.0 & 413.9 & 363.8 & 397.3 & 131.7 & 233.9 & 613.4 & 1336.7 & 685.9 & 1904.3 & 2844.0 & 1901.1 & 974.3 & 1342.1 & 3441.0 \\
\hline AMS11 & 01/04/2011 & 315.0 & 588.0 & 315.2 & 207.7 & 258.7 & 124.3 & 209.0 & 524.0 & 1455.4 & 625.5 & 1590.8 & 2536.2 & 1743.0 & 854.3 & 1180.4 & 3119.1 \\
\hline AMS11 & $30 / 04 / 2011$ & 539.0 & 971.1 & 683.0 & 581.7 & 639.0 & 361.8 & 362.8 & 1219.7 & 2572.2 & 1262.6 & 3254.9 & 6089.2 & 4495.0 & 2389.5 & 2659.2 & 7138.3 \\
\hline AMS11 & $02 / 06 / 2011$ & 38.2 & 75.8 & 73.3 & 98.6 & 124.0 & 88.1 & 54.7 & 132.1 & 379.9 & 251.5 & 336.1 & 604.2 & 501.9 & 270.8 & 226.7 & 700.4 \\
\hline AMS11 & $19 / 07 / 2011$ & 9.6 & 18.3 & 15.1 & 20.7 & 50.4 & 35.2 & 12.3 & 40.4 & 117.0 & 82.0 & 75.8 & 132.4 & 129.0 & 88.0 & 46.0 & 153.5 \\
\hline AMS11 & 02/08/2011 & 7.6 & 14.7 & 12.9 & 15.9 & 18.6 & 23.0 & 12.2 & 37.9 & 128.7 & 133.9 & 75.0 & 151.9 & 180.5 & 97.4 & 44.9 & 156.5 \\
\hline AMS11 & 01/09/2011 & 36.3 & 71.4 & 83.7 & 79.7 & 97.9 & 46.7 & 22.8 & 69.8 & 144.4 & 55.8 & 168.9 & 299.3 & 274.4 & 150.0 & 110.3 & 332.9 \\
\hline AMS11 & $30 / 09 / 2011$ & 11.9 & 23.3 & 29.4 & 32.2 & 38.6 & 25.6 & 15.7 & 38.8 & 73.6 & 57.2 & 102.0 & 194.0 & 190.1 & 97.3 & 64.0 & 190.6 \\
\hline AMS11 & $01 / 11 / 2011$ & 12.5 & 24.8 & 5.9 & 20.9 & 26.7 & 33.9 & 17.8 & 59.4 & 156.6 & 95.0 & 133.0 & 300.8 & 317.6 & 198.5 & 80.9 & 279.8 \\
\hline AMS11 & $30 / 11 / 2011$ & 5330.0 & 9490.0 & 6091.2 & 8979.8 & 8054.3 & 4326.0 & 4500.8 & $1.2 \mathrm{E}+04$ & $2.8 \mathrm{E}+04$ & $1.4 \mathrm{E}+04$ & $3.7 \mathrm{E}+04$ & $5.8 \mathrm{E}+04$ & $3.9 \mathrm{E}+04$ & $2.5 \mathrm{E}+04$ & $2.6 \mathrm{E}+04$ & $7.3 \mathrm{E}+04$ \\
\hline AMS11 & $20 / 12 / 2011$ & 168.8 & 279.7 & 163.3 & 225.5 & 230.9 & 141.6 & 162.4 & 367.4 & 779.2 & 315.8 & 1014.4 & 1460.2 & 908.8 & 450.7 & 672.6 & 1827.1 \\
\hline AMS11 & $30 / 01 / 2012$ & 356.8 & 597.9 & 580.1 & 690.6 & 607.3 & 258.5 & 383.6 & 775.9 & 1690.2 & 728.2 & 2338.2 & 3219.8 & 1917.5 & 1281.3 & 1494.2 & 4016.7 \\
\hline AMS11 & $29 / 02 / 2012$ & $1.8 \mathrm{E}+04$ & $3.0 \mathrm{E}+04$ & $3.0 \mathrm{E}+04$ & $3.7 \mathrm{E}+04$ & $3.2 \mathrm{E}+04$ & $1.6 \mathrm{E}+04$ & $1.9 \mathrm{E}+04$ & $4.2 \mathrm{E}+04$ & $9.2 \mathrm{E}+04$ & $4.1 \mathrm{E}+04$ & $1.3 \mathrm{E}+05$ & $2.0 \mathrm{E}+05$ & $1.2 \mathrm{E}+05$ & $6.9 \mathrm{E}+04$ & $8.4 \mathrm{E}+04$ & $2.2 \mathrm{E}+05$ \\
\hline AMS11 & $30 / 03 / 2012$ & 86.8 & 137.6 & 140.2 & 165.0 & 152.0 & 62.8 & 88.6 & 197.5 & 385.1 & 187.7 & 620.0 & 847.3 & 539.2 & 292.3 & 404.1 & 1094.7 \\
\hline AMS11 & $30 / 04 / 2012$ & 25.5 & 43.5 & 37.8 & 48.4 & 79.9 & 56.4 & 36.1 & 108.5 & 235.9 & 133.8 & 245.2 & 485.6 & 415.5 & 236.8 & 148.0 & 461.8 \\
\hline AMS11 & $31 / 05 / 2012$ & 4.8 & 11.4 & 34.1 & 33.4 & 45.1 & 32.8 & 13.5 & 37.9 & 73.1 & 40.6 & 56.2 & 118.0 & 156.1 & 89.8 & 29.8 & 110.0 \\
\hline
\end{tabular}


Table S1: Monthly precipitation concentrations (ng/L) cont'd

\begin{tabular}{|c|c|c|c|c|c|c|c|c|c|c|c|c|c|c|c|c|c|}
\hline Site & Start date & DahAN & BghiPER & $\begin{array}{l}\mathrm{C} 1 \\
\mathrm{NAP}\end{array}$ & $\begin{array}{c}\mathrm{C} 2 \\
\text { NAP }\end{array}$ & $\begin{array}{l}\text { C3 } \\
\text { NAP }\end{array}$ & $\begin{array}{l}\text { C4 } \\
\text { NAP }\end{array}$ & $\mathrm{C} 1 \mathrm{FL}$ & $\mathrm{C} 2 \mathrm{FL}$ & C3 FL & $\mathrm{C} 4 \mathrm{FL}$ & $\begin{array}{c}\text { C1 } \\
\text { PHE/AN }\end{array}$ & $\begin{array}{c}\mathrm{C} 2 \\
\text { PHE/AN }\end{array}$ & $\begin{array}{c}\text { C3 } \\
\text { PHE/AN }\end{array}$ & $\begin{array}{c}\text { C4 } \\
\text { PHE/AN }\end{array}$ & $\begin{array}{c}\text { C1 } \\
\text { FLT/PYR }\end{array}$ & $\begin{array}{c}\mathrm{C} 2 \\
\text { FLT/PYR }\end{array}$ \\
\hline AMS13 & $24 / 01 / 2011$ & 12.4 & 26.7 & 7.1 & 12.7 & 10.4 & 14.3 & 16.1 & 93.8 & 182.3 & 152.5 & 90.6 & 150.5 & 131.9 & 62.1 & 47.1 & 159.4 \\
\hline AMS13 & $31 / 01 / 2011$ & 46.7 & 96.5 & 130.9 & 113.1 & 57.1 & 32.0 & 59.6 & 146.4 & 366.2 & 233.4 & 417.1 & 738.9 & 775.7 & 437.5 & 263.9 & 722.4 \\
\hline AMS13 & $02 / 03 / 2011$ & 14.3 & 29.7 & 26.6 & 30.1 & 40.4 & 42.6 & 19.0 & 98.0 & 228.6 & 180.5 & 149.6 & 434.6 & 552.9 & 293.7 & 78.7 & 261.5 \\
\hline AMS13 & $01 / 04 / 2011$ & 15.2 & 33.5 & 33.2 & 38.5 & 50.2 & 55.9 & 27.4 & 114.4 & 258.9 & 186.7 & 198.1 & 512.2 & 567.6 & 321.6 & 120.2 & 404.4 \\
\hline AMS13 & $30 / 04 / 2011$ & 7.9 & 21.9 & 24.1 & 27.4 & 24.9 & 27.1 & 14.3 & 113.4 & 185.0 & 103.7 & 57.1 & 99.0 & 114.4 & 64.3 & 25.1 & 119.2 \\
\hline AMS13 & $02 / 06 / 2011$ & 1.2 & 10.3 & 8.4 & 14.9 & 32.5 & 36.9 & 6.8 & 61.5 & 109.9 & 132.2 & 71.2 & 129.2 & 133.7 & 69.0 & 42.9 & 168.9 \\
\hline AMS13 & $19 / 07 / 2011$ & 0.6 & 1.5 & 3.4 & 5.2 & 12.3 & 17.0 & 2.4 & 15.4 & 39.9 & 50.2 & 20.2 & 44.7 & 58.9 & 72.6 & 7.2 & 39.3 \\
\hline AMS13 & $02 / 08 / 2011$ & 0.3 & 0.5 & 1.4 & IDL & 3.2 & 9.3 & 1.4 & 12.3 & 19.4 & 20.7 & 6.6 & 13.3 & 21.2 & 8.2 & 1.0 & 8.8 \\
\hline AMS13 & 01/09/2011 & 0.4 & 0.5 & 2.9 & 2.7 & 8.3 & 5.2 & 2.5 & 5.2 & 11.0 & 8.4 & 10.7 & 23.9 & 34.3 & 16.8 & 3.5 & 16.9 \\
\hline AMS13 & $30 / 09 / 2011$ & 0.7 & 2.7 & 4.2 & 3.8 & 12.3 & 9.3 & 3.0 & 11.5 & 22.8 & 24.3 & 20.2 & 55.3 & 86.3 & 42.4 & 10.4 & 41.1 \\
\hline AMS13 & $01 / 11 / 2011$ & 7.8 & 15.4 & 14.1 & 17.5 & 22.3 & 20.7 & 11.4 & 30.8 & 76.8 & 44.5 & 73.8 & 115.3 & 114.7 & 82.2 & 45.8 & 133.9 \\
\hline AMS13 & $30 / 11 / 2011$ & 26.0 & 54.8 & 34.2 & 52.2 & 78.8 & 66.2 & 34.7 & 112.7 & 362.0 & 220.4 & 274.8 & 633.7 & 687.5 & 377.7 & 172.1 & 574.0 \\
\hline AMS13 & $30 / 12 / 2011$ & 17.2 & 32.0 & 22.5 & 36.6 & 47.6 & 41.0 & 27.6 & 80.1 & 165.8 & 87.2 & 152.4 & 287.5 & 247.2 & 161.7 & 85.4 & 252.4 \\
\hline AMS13 & $31 / 01 / 2012$ & 13.7 & 25.1 & IDL & 10.3 & 22.3 & 26.7 & 24.6 & 69.8 & 141.9 & 111.1 & 152.8 & 281.5 & 238.4 & 170.1 & 84.5 & 250.9 \\
\hline AMS13 & $29 / 02 / 2012$ & 197.7 & 337.3 & 218.1 & 297.0 & 471.9 & 298.7 & 262.6 & 759.3 & 1460.2 & 806.2 & 1809.2 & 3302.1 & 2694.2 & 1542.8 & 1123.9 & 3203.7 \\
\hline AMS13 & $30 / 03 / 2012$ & 24.2 & 55.2 & 57.2 & 92.6 & 189.1 & 240.3 & 114.1 & 474.7 & 987.1 & 688.1 & 725.6 & 1680.2 & 1678.4 & 955.9 & 246.7 & 960.8 \\
\hline AMS13 & $30 / 04 / 2012$ & 2.4 & 6.8 & 2.7 & 5.6 & 32.6 & 38.3 & 13.0 & 58.3 & 125.5 & 108.7 & 91.8 & 252.1 & 284.9 & 187.4 & 36.1 & 155.9 \\
\hline AMS13 & $31 / 05 / 2012$ & 3.3 & 8.4 & 35.5 & 32.1 & 40.1 & 25.8 & 6.9 & 29.5 & 54.3 & 29.9 & 48.6 & 116.1 & 165.7 & 67.9 & 22.2 & 87.6 \\
\hline
\end{tabular}


Table S1: Monthly precipitation concentrations (ng/L) cont'd

\begin{tabular}{|c|c|c|c|c|c|c|c|c|c|c|c|c|c|c|}
\hline Site & Start date & $\begin{array}{c}\text { C3 } \\
\text { FLT/PYR }\end{array}$ & $\begin{array}{c}\text { C4 } \\
\text { FLT/PYR }\end{array}$ & C1 BTC & C2 BTC & C3 BTC & C4 BTC & DBT & C1 DBT & $\mathrm{C} 2 \mathrm{DBT}$ & C3 DBT & C4 DBT & $\begin{array}{c}\text { Precip } \\
\text { amt (mm) }\end{array}$ & $\begin{array}{c}\text { Temp } \\
\left({ }^{\circ} \mathrm{C}\right)\end{array}$ \\
\hline AMS5 & $02 / 01 / 2011$ & 983.4 & 624.5 & 1175.2 & 574.9 & 418.2 & 397.1 & 55.8 & 210.6 & 327.9 & 183.4 & 109.6 & 5.7 & \\
\hline AMS5 & $31 / 01 / 2011$ & 3928.8 & 3010.7 & 4025.5 & 2649.3 & 2193.1 & 1675.4 & 352.3 & 1775.3 & 3135.4 & 2461.6 & 2077.5 & 11.8 & -11.3 \\
\hline AMS5 & $02 / 03 / 2011$ & 3952.8 & 3114.2 & 4386.4 & 2761.4 & 2048.1 & 1328.2 & 314.9 & 1453.5 & 2733.2 & 2026.2 & 1565.5 & 9.4 & -11.3 \\
\hline AMS5 & $01 / 04 / 2011$ & 3945.4 & 3218.6 & 4445.1 & 2740.9 & 2111.5 & 1587.5 & 376.0 & 1671.0 & 3107.0 & 2342.5 & 1800.0 & 9.4 & 3.2 \\
\hline AMS5 & $01 / 05 / 2011$ & 674.8 & 532.5 & 600.4 & 344.5 & 267.6 & 225.8 & 27.7 & 117.7 & 212.4 & 148.1 & 127.0 & 7.1 & 11.8 \\
\hline AMS5 & $02 / 06 / 2011$ & 128.0 & 132.7 & 81.3 & 65.6 & 62.2 & 57.4 & 7.0 & 37.3 & 77.9 & 74.6 & 86.4 & 12.3 & 14.6 \\
\hline AMS5 & $19 / 07 / 2011$ & 92.0 & 79.7 & 36.3 & 27.0 & 39.0 & 41.2 & 2.6 & 26.3 & 72.4 & 105.1 & 157.8 & 75.5 & 17.0 \\
\hline AMS5 & $02 / 08 / 2011$ & 89.6 & 63.6 & 62.5 & 42.4 & 43.7 & 36.7 & 3.3 & 20.6 & 52.2 & 61.1 & 85.4 & 13.7 & 14.3 \\
\hline AMS5 & $01 / 09 / 2011$ & 111.2 & 78.1 & 53.7 & 53.8 & 59.3 & 53.8 & 3.0 & 20.6 & 62.9 & 101.6 & 150.0 & 40.1 & 12.4 \\
\hline AMS5 & $30 / 09 / 2011$ & 76.5 & 56.4 & 57.4 & 37.3 & 40.6 & 30.6 & 3.8 & 20.7 & 51.3 & 70.4 & 89.3 & 30.2 & 2.8 \\
\hline AMS5 & $01 / 11 / 2011$ & 307.9 & 231.7 & 108.1 & 90.3 & 136.4 & 132.3 & 5.8 & 51.4 & 157.5 & 260.8 & 428.0 & 10.8 & -9.3 \\
\hline AMS5 & $30 / 11 / 2011$ & 189.0 & 133.4 & 89.7 & 63.0 & 81.5 & 82.0 & 3.9 & 36.7 & 96.9 & 112.4 & 206.4 & 2.4 & -5.2 \\
\hline AMS5 & $31 / 01 / 2012$ & 2342.3 & 1706.3 & 2240.7 & 1340.7 & 1326.2 & 930.2 & 211.5 & 988.2 & 1768.9 & 1441.5 & 1229.1 & 11.6 & -11.8 \\
\hline AMS5 & $29 / 02 / 2012$ & $4.9 \mathrm{E}+04$ & $3.5 \mathrm{E}+04$ & $5.0 \mathrm{E}+04$ & $3.3 \mathrm{E}+04$ & $2.9 \mathrm{E}+04$ & $1.9 \mathrm{E}+04$ & 3274.9 & $1.6 \mathrm{E}+04$ & $3.1 \mathrm{E}+04$ & $2.7 \mathrm{E}+04$ & $2.2 \mathrm{E}+04$ & 0.2 & -3.2 \\
\hline AMS5 & $30 / 03 / 2012$ & 660.5 & 473.4 & 653.0 & 422.5 & 388.9 & 248.9 & 58.2 & 279.0 & 503.0 & 389.9 & 342.7 & 27.8 & 0.2 \\
\hline AMS5 & $30 / 04 / 2012$ & 297.7 & 220.0 & 207.0 & 162.2 & 185.0 & 134.6 & 13.4 & 82.3 & 213.2 & 249.8 & 318.5 & 21.2 & 11.3 \\
\hline AMS5 & $31 / 05 / 2012$ & 430.3 & 287.2 & 226.4 & 207.3 & 241.8 & 220.4 & 14.9 & 91.0 & 244.4 & 324.0 & 485.1 & 16.5 & 14.6 \\
\hline
\end{tabular}


Table S1: Monthly precipitation concentrations (ng/L) cont'd

\begin{tabular}{|c|c|c|c|c|c|c|c|c|c|c|c|c|c|c|}
\hline Site & Start date & $\begin{array}{c}\text { C3 } \\
\text { FLT/PYR }\end{array}$ & $\begin{array}{c}\text { C4 } \\
\text { FLT/PYR }\end{array}$ & C1 BTC & $\mathrm{C} 2 \mathrm{BTC}$ & C3 BTC & C4 BTC & DBT & C1 DBT & C2 DBT & C3 DBT & C4 DBT & $\begin{array}{l}\text { Precip amt } \\
(\mathrm{mm})\end{array}$ & $\begin{array}{c}\text { Temp } \\
\left({ }^{\circ} \mathrm{C}\right)\end{array}$ \\
\hline AMS11 & 02/01/2011 & 2662.3 & 1622.6 & 2612.1 & 1605.3 & 1315.5 & 864.0 & 191.3 & 921.8 & 1762.1 & 1410.3 & 1206.4 & 3.5 & -17.4 \\
\hline AMS11 & $31 / 01 / 2011$ & 6615.6 & 5115.4 & 6847.1 & 4296.2 & 3746.7 & 2170.3 & 504.0 & 2293.9 & 3760.9 & 2842.8 & 2108.3 & 7.1 & -9.5 \\
\hline AMS11 & $02 / 03 / 2011$ & 4440.7 & 3191.6 & 5237.6 & 3112.5 & 2612.5 & 1635.0 & 408.2 & 1820.6 & 3231.2 & 2304.9 & 1731.8 & 7.1 & -11.3 \\
\hline AMS11 & $01 / 04 / 2011$ & 4038.1 & 3375.3 & 4738.9 & 2993.8 & 2617.5 & 1615.0 & 334.6 & 1471.7 & 2788.2 & 1977.5 & 1544.6 & 4.7 & 3.2 \\
\hline AMS11 & $30 / 04 / 2011$ & 9598.0 & 6658.1 & $1.1 \mathrm{E}+04$ & 6744.2 & 5639.4 & 4020.2 & 720.4 & 3142.8 & 6739.9 & 5353.8 & 4491.2 & 1.9 & 12.3 \\
\hline AMS11 & 02/06/2011 & 941.4 & 760.2 & 824.3 & 563.1 & 542.5 & 377.6 & 61.5 & 322.1 & 631.0 & 542.5 & 538.6 & 9.4 & 14.9 \\
\hline AMS11 & $19 / 07 / 2011$ & 222.5 & 168.1 & 173.8 & 113.3 & 95.8 & 65.4 & 15.0 & 73.5 & 145.8 & 141.2 & 175.9 & 62.5 & 17.5 \\
\hline AMS11 & 02/08/2011 & 254.8 & 183.2 & 164.1 & 119.5 & 115.2 & 83.9 & 12.3 & 56.0 & 127.3 & 160.7 & 213.0 & 23.6 & 14.4 \\
\hline AMS11 & $01 / 09 / 2011$ & 455.9 & 333.4 & 389.0 & 272.6 & 267.3 & 163.3 & 27.7 & 124.5 & 257.6 & 264.2 & 302.3 & 27.8 & 12.4 \\
\hline AMS11 & $30 / 09 / 2011$ & 255.2 & 186.8 & 232.2 & 168.0 & 144.4 & 114.5 & 16.4 & 85.0 & 182.8 & 186.8 & 207.0 & 27.4 & 2.8 \\
\hline AMS11 & $01 / 11 / 2011$ & 417.2 & 317.2 & 280.2 & 198.0 & 208.7 & 167.2 & 16.5 & 101.4 & 254.7 & 301.9 & 412.0 & 10.6 & -9.3 \\
\hline AMS11 & $30 / 11 / 2011$ & $9.3 \mathrm{E}+04$ & $6.7 \mathrm{E}+04$ & $9.6 \mathrm{E}+04$ & $6.2 \mathrm{E}+04$ & $5.2 \mathrm{E}+04$ & $3.7 \mathrm{E}+04$ & 6244.3 & $3.1 \mathrm{E}+04$ & $6.1 \mathrm{E}+04$ & $5.1 \mathrm{E}+04$ & $5.4 \mathrm{E}+04$ & 0.0 & -5.2 \\
\hline AMS11 & $20 / 12 / 2011$ & 2215.7 & 1642.4 & 2438.5 & 1540.8 & 1189.1 & 740.6 & 190.2 & 978.6 & 1804.4 & 1365.3 & 1116.8 & 5.7 & -14.6 \\
\hline AMS11 & $30 / 01 / 2012$ & 5131.0 & 3666.7 & 5229.3 & 3100.5 & 2985.2 & 1923.8 & 515.1 & 2356.7 & 4093.4 & 3110.8 & 2503.4 & 7.1 & -11.8 \\
\hline AMS11 & $29 / 02 / 2012$ & $3.0 \mathrm{E}+05$ & $2.2 \mathrm{E}+05$ & $3.0 \mathrm{E}+05$ & $2.0 \mathrm{E}+05$ & $1.6 \mathrm{E}+05$ & $1.2 \mathrm{E}+05$ & $2.8 \mathrm{E}+04$ & $1.3 \mathrm{E}+05$ & $2.3 \mathrm{E}+05$ & $1.8 \mathrm{E}+05$ & $1.5 \mathrm{E}+05$ & 0.1 & -2.3 \\
\hline AMS11 & $30 / 03 / 2012$ & 1393.6 & 982.8 & 1425.5 & 915.4 & 783.6 & 483.7 & 117.4 & 563.1 & 1001.7 & 773.5 & 643.0 & 20.0 & -0.9 \\
\hline AMS11 & $30 / 04 / 2012$ & 646.7 & 460.1 & 540.5 & 388.9 & 384.3 & 274.5 & 37.7 & 204.2 & 469.3 & 485.8 & 530.5 & 17.9 & 10.5 \\
\hline AMS11 & $31 / 05 / 2012$ & 168.2 & 115.7 & 105.3 & 80.5 & 98.4 & 82.7 & 6.3 & 39.5 & 93.4 & 114.9 & 162.2 & 21.7 & 15.0 \\
\hline
\end{tabular}


Table S1: Monthly precipitation concentrations (ng/L) cont'd

\begin{tabular}{|c|c|c|c|c|c|c|c|c|c|c|c|c|c|c|}
\hline Site & Start date & $\begin{array}{c}\text { C3 } \\
\text { FLT/PYR }\end{array}$ & $\begin{array}{c}\text { C4 } \\
\text { FLT/PYR }\end{array}$ & C1 BTC & C2 BTC & С3 BTC & C4 BTC & DBT & C1 DBT & $\mathrm{C} 2 \mathrm{DBT}$ & C3 DBT & C4 DBT & $\begin{array}{c}\text { Precip } \\
\text { amt }(\mathrm{mm})\end{array}$ & $\begin{array}{l}\text { Temp } \\
\left({ }^{\circ} \mathrm{C}\right)\end{array}$ \\
\hline AMS13 & $24 / 01 / 2011$ & 263.6 & 201.0 & 213.9 & 133.5 & 126.3 & 76.5 & 9.5 & 47.2 & 102.0 & 97.2 & 81.4 & 21.2 & -16.8 \\
\hline AMS13 & $31 / 01 / 2011$ & 1067.3 & 690.3 & 974.8 & 682.7 & 671.5 & 494.4 & 66.6 & 332.5 & 713.0 & 703.1 & 703.3 & 4.7 & -9.5 \\
\hline AMS13 & $02 / 03 / 2011$ & 417.1 & 296.2 & 227.0 & 184.8 & 236.4 & 171.6 & 9.3 & 61.6 & 231.8 & 344.2 & 437.8 & 15.3 & -11.3 \\
\hline AMS13 & $01 / 04 / 2011$ & 695.7 & 527.2 & 375.4 & 326.2 & 431.5 & 362.1 & 17.8 & 110.5 & 345.3 & 471.6 & 638.4 & 9.4 & 3.2 \\
\hline AMS13 & $30 / 04 / 2011$ & 263.6 & 236.9 & 97.6 & 77.3 & 95.6 & 74.2 & 2.5 & 18.3 & 51.5 & 48.3 & 74.9 & 9.0 & 11.8 \\
\hline AMS13 & $02 / 06 / 2011$ & 265.4 & 233.2 & 154.7 & 108.6 & 121.6 & 89.3 & 9.3 & 54.1 & 130.5 & 138.3 & 176.3 & 6.1 & 14.6 \\
\hline AMS13 & $19 / 07 / 2011$ & 56.9 & 47.6 & 22.2 & 19.0 & 24.2 & 23.4 & 1.5 & 11.9 & 34.5 & 43.5 & 109.1 & 93.8 & 17.3 \\
\hline AMS13 & $02 / 08 / 2011$ & 14.9 & 15.2 & 4.9 & 4.1 & 6.1 & 0.3 & 0.1 & 1.5 & 10.9 & 13.7 & 23.8 & 23.6 & 14.4 \\
\hline AMS13 & $01 / 09 / 2011$ & 26.5 & 18.6 & 11.8 & 12.1 & 15.4 & 13.6 & 0.6 & 4.3 & 14.0 & 22.0 & 32.5 & 40.1 & 12.4 \\
\hline AMS13 & $30 / 09 / 2011$ & 59.9 & 34.5 & 31.4 & 23.9 & 29.4 & 26.3 & 1.5 & 9.6 & 32.9 & 55.6 & 76.5 & 19.8 & 2.8 \\
\hline AMS13 & $01 / 11 / 2011$ & 179.9 & 132.8 & 154.8 & 93.6 & 103.3 & 73.3 & 9.7 & 49.5 & 117.4 & 112.2 & 133.1 & 13.2 & -9.3 \\
\hline AMS13 & $30 / 11 / 2011$ & 878.7 & 643.0 & 561.4 & 423.0 & 427.4 & 373.6 & 27.5 & 177.5 & 479.5 & 631.3 & 780.5 & 4.7 & -5.2 \\
\hline AMS13 & $30 / 12 / 2011$ & 349.3 & 269.5 & 294.1 & 202.0 & 194.5 & 154.4 & 16.8 & 95.6 & 228.2 & 244.1 & 270.2 & 13.0 & -16.0 \\
\hline AMS13 & $31 / 01 / 2012$ & 355.9 & 247.8 & 294.6 & 186.8 & 191.7 & 142.7 & 20.5 & 104.8 & 226.9 & 213.5 & 244.8 & 11.8 & -11.8 \\
\hline AMS13 & $29 / 02 / 2012$ & 4492.3 & 3165.4 & 4026.5 & 2743.6 & 2474.4 & 1614.5 & 271.4 & 1420.4 & 3102.2 & 2929.2 & 3122.8 & 1.4 & 1.3 \\
\hline AMS13 & $30 / 03 / 2012$ & 1623.4 & 1080.7 & 798.8 & 678.4 & 828.2 & 683.2 & 44.9 & 342.5 & 1173.6 & 1661.2 & 2207.4 & 4.7 & 0.2 \\
\hline AMS13 & $30 / 04 / 2012$ & 277.0 & 193.5 & 109.0 & 112.3 & 147.0 & 122.0 & 4.3 & 41.3 & 165.8 & 267.5 & 404.9 & 20.0 & 11.3 \\
\hline AMS13 & $31 / 05 / 2012$ & 119.6 & 88.8 & 79.6 & 60.3 & 76.8 & 65.1 & 3.8 & 26.8 & 86.1 & 109.8 & 145.4 & 16.5 & 14.6 \\
\hline
\end{tabular}


Table S2: Monthly average air concentrations $\left(\mathrm{ng} / \mathrm{m}^{3}\right)$ based on 4-5 weekly air concentrations

\begin{tabular}{|c|c|c|c|c|c|c|c|c|c|c|c|c|c|c|c|c|c|}
\hline Site & Start date & NAP & $\mathrm{AL}$ & $\mathrm{AE}$ & $\mathrm{FL}$ & $\mathrm{PHE}$ & AN & FLT & PY & RET & $\mathrm{BaAN}$ & $\mathrm{CHR}$ & $\mathrm{BbFLT}$ & BkFLT & BaPY & PER & IP \\
\hline AMS5 & 02/01/2011 & - & - & - & - & - & - & - & - & - & - & - & - & - & - & - & - \\
\hline AMS5 & $31 / 01 / 2011$ & 8.5 & 0.6 & 0.8 & 0.9 & 2.1 & 0.2 & 0.5 & 0.7 & 0.8 & 0.1 & 0.3 & 0.2 & $3.4 \mathrm{E}-02$ & 0.1 & $1.4 \mathrm{E}-02$ & $6.4 \mathrm{E}-02$ \\
\hline AMS5 & 02/03/2011 & 8.6 & 1.0 & 1.0 & 1.0 & 1.5 & 0.1 & 0.4 & 0.5 & 1.3 & 0.2 & 0.4 & 0.2 & $5.1 \mathrm{E}-02$ & 0.2 & $1.8 \mathrm{E}-02$ & $8.6 \mathrm{E}-02$ \\
\hline AMS5 & 01/04/2011 & 1.8 & 0.2 & 0.7 & 0.8 & 2.2 & 0.2 & 0.2 & 0.3 & 0.1 & 5.7E-02 & 0.2 & 4.9E-02 & $7.8 \mathrm{E}-03$ & $5.3 \mathrm{E}-02$ & $3.2 \mathrm{E}-03$ & $1.7 \mathrm{E}-02$ \\
\hline AMS5 & $01 / 05 / 2011$ & 1.2 & 0.2 & 0.6 & 1.7 & 11.5 & 0.8 & 1.6 & 1.5 & 97.3 & 0.5 & 0.9 & 0.4 & 0.1 & 0.3 & $2.4 \mathrm{E}-02$ & 0.2 \\
\hline AMS5 & $02 / 06 / 2011$ & 0.5 & 0.2 & 0.8 & 1.5 & 5.6 & 0.3 & 0.5 & 0.5 & 11.3 & 0.2 & 0.3 & 0.1 & $3.3 \mathrm{E}-02$ & 0.1 & $1.5 \mathrm{E}-02$ & $5.8 \mathrm{E}-02$ \\
\hline AMS5 & $19 / 07 / 2011$ & NA & $7.2 \mathrm{E}-02$ & 0.6 & 0.5 & 2.2 & 0.2 & 0.2 & 0.3 & 0.5 & $1.2 \mathrm{E}-02$ & $3.6 \mathrm{E}-02$ & 1.4E-02 & $5.4 \mathrm{E}-05$ & $9.4 \mathrm{E}-03$ & $6.2 \mathrm{E}-05$ & $3.9 \mathrm{E}-03$ \\
\hline AMS5 & $02 / 08 / 2011$ & 0.3 & 0.1 & 0.5 & 0.4 & 1.7 & 7.9E-02 & 0.1 & 0.2 & 0.2 & 8.9E-03 & $2.5 \mathrm{E}-02$ & $9.6 \mathrm{E}-03$ & 3.7E-05 & $9.3 \mathrm{E}-03$ & $2.2 \mathrm{E}-03$ & $1.6 \mathrm{E}-03$ \\
\hline AMS5 & 01/09/2011 & 0.7 & 0.2 & 0.5 & 0.4 & 1.2 & 8.5E-02 & 0.1 & 0.2 & 0.2 & 2.0E-02 & 4.7E-02 & $3.3 \mathrm{E}-02$ & $6.3 \mathrm{E}-03$ & $2.1 \mathrm{E}-02$ & $3.1 \mathrm{E}-03$ & $1.7 \mathrm{E}-02$ \\
\hline AMS5 & $30 / 09 / 2011$ & 0.4 & 0.3 & 0.3 & 0.7 & 1.4 & 0.2 & 0.2 & 0.3 & 0.2 & 4.3E-02 & $6.7 \mathrm{E}-02$ & $4.8 \mathrm{E}-02$ & 1.6E-02 & $3.0 \mathrm{E}-02$ & $8.8 \mathrm{E}-03$ & $3.4 \mathrm{E}-02$ \\
\hline AMS5 & 01/11/2011 & 3.2 & 1.1 & 0.7 & 0.9 & 1.8 & 0.2 & 0.5 & 0.6 & 0.5 & 0.2 & 0.4 & 0.2 & 4.6E-02 & 0.2 & 1.9E-02 & $8.1 \mathrm{E}-02$ \\
\hline AMS5 & $30 / 11 / 2011$ & 1.7 & 0.6 & 0.5 & 1.1 & 2.0 & 0.1 & 0.4 & 0.4 & 0.6 & 4.7E-02 & $9.2 \mathrm{E}-02$ & 8.9E-02 & 2.7E-02 & $3.5 \mathrm{E}-02$ & $6.5 \mathrm{E}-03$ & $5.0 \mathrm{E}-02$ \\
\hline AMS5 & $30 / 12 / 2011$ & 13.4 & 1.3 & 1.1 & 1.3 & 2.5 & 0.2 & 0.6 & 0.6 & 2.1 & 0.1 & 0.3 & 0.2 & 4.1E-02 & 9.3E-02 & 1.4E-02 & $6.9 \mathrm{E}-02$ \\
\hline AMS5 & $31 / 01 / 2012$ & 13.8 & 1.9 & 1.4 & 1.8 & 4.6 & 0.5 & 1.3 & 1.7 & 2.9 & 0.6 & 1.0 & 0.3 & 0.1 & 0.6 & $5.9 \mathrm{E}-02$ & 0.2 \\
\hline AMS5 & $29 / 02 / 2012$ & 3.0 & 0.5 & 1.3 & 1.1 & 2.6 & 0.3 & 0.4 & 0.8 & 0.1 & 0.5 & 0.7 & 0.2 & $5.4 \mathrm{E}-02$ & 0.5 & 4.2E-02 & 0.1 \\
\hline AMS5 & $30 / 03 / 2012$ & 1.0 & 0.2 & 0.5 & 0.6 & 1.3 & $5.2 \mathrm{E}-02$ & 0.2 & 0.3 & $8.8 \mathrm{E}-02$ & $6.1 \mathrm{E}-02$ & 0.1 & $5.8 \mathrm{E}-02$ & 1.3E-02 & 7.0E-02 & $2.3 \mathrm{E}-02$ & $2.7 \mathrm{E}-02$ \\
\hline AMS5 & $30 / 04 / 2012$ & 0.6 & $9.0 \mathrm{E}-02$ & 0.4 & 0.6 & 1.6 & 5.9E-02 & 0.2 & 0.3 & 0.1 & $5.8 \mathrm{E}-02$ & 0.1 & 5.3E-02 & 6.9E-03 & 6.7E-02 & 1.6E-02 & $2.4 \mathrm{E}-02$ \\
\hline AMS5 & $31 / 05 / 2012$ & 0.6 & 7.9E-02 & 0.5 & 0.7 & 3.9 & 0.2 & 0.3 & 0.5 & 3.1 & $9.5 \mathrm{E}-02$ & 0.3 & 0.1 & $2.0 \mathrm{E}-02$ & $9.4 \mathrm{E}-02$ & $1.8 \mathrm{E}-02$ & $5.3 \mathrm{E}-02$ \\
\hline
\end{tabular}


Table S2: Monthly average air concentrations $\left(\mathrm{ng} / \mathrm{m}^{3}\right)$ based on 4-5 weekly air concentrations cont'd

\begin{tabular}{|c|c|c|c|c|c|c|c|c|c|c|c|c|c|c|c|c|c|}
\hline Site & Start date & NAP & $\mathrm{AL}$ & $\mathrm{AE}$ & $\mathrm{FL}$ & PHE & AN & FLT & PY & RET & $\mathrm{BaAN}$ & $\mathrm{CHR}$ & $\mathrm{BbFLT}$ & BkFLT & BaPY & PER & IP \\
\hline AMS11 & 02/01/2011 & 7.5 & 0.9 & 1.5 & 1.1 & 3.1 & 0.3 & 0.4 & 1.0 & 0.6 & 0.5 & 0.9 & 0.4 & 6.7E-02 & 0.5 & 7.1E-02 & 0.1 \\
\hline AMS11 & $31 / 01 / 2011$ & 4.6 & 0.3 & 0.8 & 0.8 & 2.2 & 0.2 & 0.4 & 0.6 & 1.2 & 0.2 & 0.4 & 0.2 & 4.6E-02 & 0.2 & $1.9 \mathrm{E}-02$ & 7.4E-02 \\
\hline AMS11 & $02 / 03 / 2011$ & 4.5 & 0.4 & 0.9 & 0.9 & 3.0 & 0.2 & 0.4 & 0.5 & 1.1 & 0.3 & 0.5 & 0.2 & $5.6 \mathrm{E}-02$ & 0.3 & $1.6 \mathrm{E}-02$ & 8.4E-02 \\
\hline AMS11 & $01 / 04 / 2011$ & 1.2 & $9.9 \mathrm{E}-02$ & 0.8 & 0.2 & 1.0 & 0.2 & 5.7E-02 & 0.3 & $3.4 \mathrm{E}-02$ & 0.3 & 0.4 & 0.1 & 3.7E-02 & 0.2 & $1.6 \mathrm{E}-05$ & 3.7E-02 \\
\hline AMS11 & $30 / 04 / 2011$ & 2.2 & 0.2 & 1.0 & 1.6 & 13.6 & 1.3 & 1.6 & 2.0 & 82.1 & 1.9 & 2.3 & 1.4 & 0.4 & 1.4 & 0.1 & 0.4 \\
\hline AMS11 & $02 / 06 / 2011$ & 1.0 & 0.2 & 0.6 & 1.6 & 4.8 & 0.3 & 0.5 & 0.5 & 11.9 & 0.2 & 0.5 & 0.2 & $5.4 \mathrm{E}-02$ & 0.2 & $2.0 \mathrm{E}-02$ & 0.1 \\
\hline AMS11 & $19 / 07 / 2011$ & NA & 4.1E-02 & 0.7 & 0.5 & 2.9 & 0.2 & 0.2 & 0.4 & 0.3 & $5.2 \mathrm{E}-02$ & $9.9 \mathrm{E}-02$ & $2.5 \mathrm{E}-02$ & $5.2 \mathrm{E}-03$ & 4.4E-02 & 7.5E-03 & $1.0 \mathrm{E}-02$ \\
\hline AMS11 & $02 / 08 / 2011$ & 0.8 & 0.1 & 0.9 & 0.7 & 2.4 & 0.1 & 0.1 & 0.2 & 0.2 & $2.5 \mathrm{E}-02$ & $8.2 \mathrm{E}-02$ & 3.7E-02 & 7.2E-03 & $2.2 \mathrm{E}-02$ & $5.5 \mathrm{E}-03$ & $1.1 \mathrm{E}-02$ \\
\hline AMS11 & 01/09/2011 & 0.4 & 0.1 & 0.7 & 0.7 & 2.4 & 0.3 & 0.1 & 0.3 & 0.2 & 7.1E-02 & $9.6 \mathrm{E}-02$ & $2.9 \mathrm{E}-02$ & 8.4E-03 & $4.8 \mathrm{E}-02$ & $1.2 \mathrm{E}-02$ & $1.6 \mathrm{E}-02$ \\
\hline AMS11 & $30 / 09 / 2011$ & 1.0 & 0.1 & 0.6 & 0.6 & 1.5 & 0.2 & 0.1 & 0.3 & 0.2 & 0.3 & 0.5 & 0.1 & $3.5 \mathrm{E}-02$ & 0.3 & $2.0 \mathrm{E}-02$ & $5.8 \mathrm{E}-02$ \\
\hline AMS11 & $01 / 11 / 2011$ & 3.5 & 0.4 & 0.7 & 0.8 & 2.6 & 0.7 & 0.5 & 1.5 & 0.7 & 1.4 & 2.0 & 0.6 & 0.2 & 1.4 & 0.1 & 0.4 \\
\hline AMS11 & $30 / 11 / 2011$ & 2.3 & 0.4 & 2.4 & 1.3 & 3.7 & 0.5 & 0.5 & 1.3 & 0.7 & 1.1 & 1.8 & 0.4 & 0.1 & 1.1 & 8.7E-02 & 0.3 \\
\hline AMS11 & $20 / 12 / 2011$ & 10.6 & 0.5 & 1.4 & 1.1 & 2.6 & 0.3 & 0.4 & 0.8 & 1.1 & 0.6 & 1.1 & 0.3 & $9.0 \mathrm{E}-02$ & 0.7 & $4.8 \mathrm{E}-02$ & 0.2 \\
\hline AMS11 & $30 / 01 / 2012$ & 10.1 & 0.4 & 1.9 & 1.5 & 4.9 & 0.8 & 0.9 & 2.3 & 1.9 & 2.3 & 3.3 & 1.0 & 0.3 & 2.7 & 0.2 & 0.7 \\
\hline AMS11 & $29 / 02 / 2012$ & 1.4 & 0.1 & 1.1 & 0.9 & 2.2 & 0.1 & 0.2 & 0.4 & 0.1 & 0.3 & 0.5 & 0.1 & $3.6 \mathrm{E}-02$ & 0.4 & $3.8 \mathrm{E}-02$ & 7.9E-02 \\
\hline AMS11 & $30 / 03 / 2012$ & 1.0 & $6.3 \mathrm{E}-02$ & 0.7 & 0.8 & 2.4 & 0.2 & 0.2 & 0.4 & $6.9 \mathrm{E}-02$ & 0.2 & 0.4 & 0.1 & $3.8 \mathrm{E}-02$ & 0.3 & $5.6 \mathrm{E}-02$ & $6.9 \mathrm{E}-02$ \\
\hline AMS11 & $30 / 04 / 2012$ & 0.5 & 3.0E-02 & 0.6 & 0.8 & 2.6 & $9.3 \mathrm{E}-02$ & 0.1 & 0.2 & 7.6E-02 & 0.1 & 0.2 & $5.9 \mathrm{E}-02$ & 1.4E-02 & 0.1 & $2.4 \mathrm{E}-02$ & $3.5 \mathrm{E}-02$ \\
\hline AMS11 & $31 / 05 / 2012$ & 0.6 & $1.6 \mathrm{E}-02$ & 0.3 & 0.8 & 3.5 & 0.1 & 0.3 & 0.4 & 4.3 & 0.2 & 0.4 & 0.2 & 4.3E-02 & 0.2 & 2.7E-02 & $9.5 \mathrm{E}-02$ \\
\hline
\end{tabular}


Table S2: Monthly average air concentrations $\left(\mathrm{ng} / \mathrm{m}^{3}\right)$ based on 4-5 weekly air concentrations cont'd

\begin{tabular}{|c|c|c|c|c|c|c|c|c|c|c|c|c|c|c|c|c|c|}
\hline Site & Start date & NAP & $\mathrm{AL}$ & $\mathrm{AE}$ & $\mathrm{FL}$ & PHE & AN & FLT & PY & RET & $\mathrm{BaAN}$ & $\mathrm{CHR}$ & $\mathrm{BbFLT}$ & BkFLT & $\mathrm{BaPY}$ & PER & IP \\
\hline AMS13 & $24 / 01 / 2011$ & 2.2 & 0.7 & 1.3 & 1.3 & 2.1 & 0.1 & 0.2 & 0.2 & 1.8 & $5.5 \mathrm{E}-02$ & 0.1 & 0.1 & $2.2 \mathrm{E}-02$ & $4.3 \mathrm{E}-02$ & 3.7E-04 & $3.9 \mathrm{E}-02$ \\
\hline AMS13 & $31 / 01 / 2011$ & 3.5 & 0.2 & 0.5 & 0.6 & 1.2 & $2.8 \mathrm{E}-02$ & 0.2 & 0.2 & 0.6 & $5.4 \mathrm{E}-02$ & 0.1 & $8.4 \mathrm{E}-02$ & $2.8 \mathrm{E}-02$ & $3.3 \mathrm{E}-02$ & $2.8 \mathrm{E}-03$ & $5.0 \mathrm{E}-02$ \\
\hline AMS13 & $02 / 03 / 2011$ & 1.2 & 0.2 & 0.4 & 0.6 & 1.6 & 5.3E-02 & 0.3 & 0.3 & 1.7 & 7.1E-02 & 0.2 & 0.1 & 3.1E-02 & $6.4 \mathrm{E}-02$ & $7.8 \mathrm{E}-03$ & 4.7E-02 \\
\hline AMS13 & 01/04/2011 & 0.7 & 0.1 & 1.2 & 0.6 & 1.4 & $2.4 \mathrm{E}-02$ & $5.1 \mathrm{E}-02$ & $5.0 \mathrm{E}-02$ & $7.4 \mathrm{E}-02$ & 2.1E-03 & 1.7E-02 & 7.5E-03 & $5.0 \mathrm{E}-03$ & $2.3 \mathrm{E}-03$ & $9.9 \mathrm{E}-04$ & $2.3 \mathrm{E}-03$ \\
\hline AMS13 & $30 / 04 / 2011$ & 3.4 & 0.3 & 1.0 & 5.2 & 14.3 & 0.5 & 2.0 & 1.3 & 141.3 & 0.6 & 1.5 & 0.8 & 8.9E-02 & 0.5 & $3.6 \mathrm{E}-02$ & 0.6 \\
\hline AMS13 & $02 / 06 / 2011$ & 1.2 & 0.2 & 0.3 & 1.9 & 5.3 & 0.2 & 0.5 & 0.3 & 20.5 & 0.1 & 0.4 & 0.3 & $3.2 \mathrm{E}-02$ & $9.9 \mathrm{E}-02$ & $1.2 \mathrm{E}-02$ & 0.1 \\
\hline AMS13 & $19 / 07 / 2011$ & NA & $1.5 \mathrm{E}-02$ & $5.8 \mathrm{E}-02$ & 0.2 & 0.7 & $2.9 \mathrm{E}-02$ & 3.7E-02 & $4.9 \mathrm{E}-02$ & 0.5 & NA & NA & NA & 7.9E-06 & NA & $1.1 \mathrm{E}-03$ & NA \\
\hline AMS13 & 02/08/2011 & NA & 5.7E-03 & $6.2 \mathrm{E}-02$ & 0.2 & 0.5 & $9.1 \mathrm{E}-03$ & $2.9 \mathrm{E}-02$ & $3.8 \mathrm{E}-02$ & 0.2 & NA & NA & NA & 2.7E-05 & NA & $1.0 \mathrm{E}-03$ & NA \\
\hline AMS13 & 01/09/2011 & NA & 0.2 & 8.1E-02 & 0.2 & 0.4 & $3.6 \mathrm{E}-02$ & $2.9 \mathrm{E}-02$ & 4.1E-02 & 0.2 & $8.4 \mathrm{E}-03$ & $2.9 \mathrm{E}-02$ & $6.0 \mathrm{E}-02$ & 4.2E-03 & $8.4 \mathrm{E}-03$ & $9.3 \mathrm{E}-04$ & $2.8 \mathrm{E}-02$ \\
\hline AMS13 & $30 / 09 / 2011$ & $1.4 \mathrm{E}-02$ & $3.4 \mathrm{E}-02$ & 0.2 & 0.3 & 0.5 & $2.2 \mathrm{E}-02$ & $3.1 \mathrm{E}-02$ & 4.0E-02 & 0.2 & 1.7E-02 & $2.9 \mathrm{E}-02$ & 7.9E-03 & $2.9 \mathrm{E}-03$ & $1.5 \mathrm{E}-02$ & $2.2 \mathrm{E}-03$ & $8.4 \mathrm{E}-03$ \\
\hline AMS13 & $01 / 11 / 2011$ & 1.7 & 0.3 & 0.4 & 0.4 & 0.6 & 2.7E-02 & 0.1 & 0.1 & 0.3 & $2.4 \mathrm{E}-02$ & $6.3 \mathrm{E}-02$ & 5.7E-02 & $1.9 \mathrm{E}-02$ & $2.0 \mathrm{E}-02$ & $7.3 \mathrm{E}-03$ & $3.4 \mathrm{E}-02$ \\
\hline AMS13 & $30 / 11 / 2011$ & 1.4 & 0.3 & 0.6 & 0.7 & 1.0 & 4.9E-02 & 0.2 & 0.2 & 0.4 & 4.5E-02 & $8.9 \mathrm{E}-02$ & $6.4 \mathrm{E}-02$ & 1.7E-02 & 4.2E-02 & 3.0E-03 & $3.2 \mathrm{E}-02$ \\
\hline AMS13 & $30 / 12 / 2011$ & 8.8 & 0.4 & 0.4 & 0.6 & 1.0 & 4.3E-02 & 0.3 & 0.2 & 1.7 & $5.8 \mathrm{E}-02$ & 0.1 & 0.1 & 4.0E-02 & 5.7E-02 & 4.1E-03 & $4.9 \mathrm{E}-02$ \\
\hline AMS13 & $31 / 01 / 2012$ & 5.4 & 0.2 & 0.9 & 0.7 & 1.4 & $9.5 \mathrm{E}-02$ & 0.3 & 0.3 & 0.9 & 0.2 & 0.3 & 0.1 & 3.7E-02 & 0.2 & $1.9 \mathrm{E}-02$ & 6.7E-02 \\
\hline AMS13 & 29/02/2012 & 0.7 & 4.6E-02 & 0.7 & 0.7 & 1.2 & 1.7E-02 & $6.0 \mathrm{E}-02$ & $5.6 \mathrm{E}-02$ & 4.3E-02 & $2.3 \mathrm{E}-02$ & $5.4 \mathrm{E}-02$ & $1.1 \mathrm{E}-02$ & 2.0E-04 & $2.9 \mathrm{E}-02$ & 7.3E-03 & $3.5 \mathrm{E}-03$ \\
\hline AMS13 & $30 / 03 / 2012$ & 1.0 & $3.2 \mathrm{E}-02$ & 0.4 & 0.4 & 0.8 & $1.1 \mathrm{E}-02$ & $3.8 \mathrm{E}-02$ & 4.6E-02 & $2.9 \mathrm{E}-02$ & $1.2 \mathrm{E}-02$ & $2.6 \mathrm{E}-02$ & $1.4 \mathrm{E}-02$ & $9.5 \mathrm{E}-03$ & $1.9 \mathrm{E}-02$ & $6.6 \mathrm{E}-03$ & $9.4 \mathrm{E}-03$ \\
\hline AMS13 & $30 / 04 / 2012$ & 0.2 & $7.4 \mathrm{E}-03$ & 7.7E-02 & 0.3 & 0.9 & $3.8 \mathrm{E}-02$ & 4.3E-02 & $8.0 \mathrm{E}-02$ & $3.5 \mathrm{E}-02$ & $3.8 \mathrm{E}-02$ & $6.8 \mathrm{E}-02$ & $2.1 \mathrm{E}-02$ & 4.3E-03 & $5.2 \mathrm{E}-02$ & 8.1E-03 & $1.3 \mathrm{E}-02$ \\
\hline AMS13 & $31 / 05 / 2012$ & 0.2 & $1.2 \mathrm{E}-02$ & 0.2 & 0.5 & 2.1 & $2.2 \mathrm{E}-02$ & 0.1 & $9.1 \mathrm{E}-02$ & 2.5 & $1.4 \mathrm{E}-02$ & $5.6 \mathrm{E}-02$ & $5.6 \mathrm{E}-02$ & $1.5 \mathrm{E}-02$ & $7.6 \mathrm{E}-03$ & $0.0 \mathrm{E}+00$ & $2.6 \mathrm{E}-02$ \\
\hline
\end{tabular}


Table S2: Monthly average air concentrations $\left(\mathrm{ng} / \mathrm{m}^{3}\right)$ based on 4-5 weekly air concentrations cont'd

\begin{tabular}{|c|c|c|c|c|c|c|c|c|c|c|c|c|c|c|c|c|c|}
\hline Site & Start date & DahAN & BghiPER & C1 NAP & C2 NAP & C3 NAP & C4 NAP & $\mathrm{C} 1 \mathrm{FL}$ & $\mathrm{C} 2 \mathrm{FL}$ & C3 FL & $\mathrm{C} 4 \mathrm{FL}$ & $\begin{array}{c}\text { C1 } \\
\text { PHE/AN }\end{array}$ & $\begin{array}{c}\mathrm{C} 2 \\
\mathrm{PHE} / \mathrm{AN}\end{array}$ & $\begin{array}{c}\text { C3 } \\
\text { PHE/AN }\end{array}$ & $\begin{array}{c}\mathrm{C} 4 \\
\text { PHE/AN }\end{array}$ & $\begin{array}{c}\text { C1 } \\
\text { FLT/PYR }\end{array}$ & $\begin{array}{c}\text { C2 } \\
\text { FLT/PYR }\end{array}$ \\
\hline AMS5 & 02/01/2011 & - & - & - & - & - & - & - & - & - & - & - & - & - & - & - & - \\
\hline AMS5 & $31 / 01 / 2011$ & $3.3 \mathrm{E}-02$ & 0.1 & 11.8 & 14.7 & 11.9 & 6.2 & 1.8 & 3.0 & 2.9 & 1.5 & 2.1 & 2.5 & 2.4 & 1.6 & 0.5 & 1.1 \\
\hline AMS5 & 02/03/2011 & 5.7E-02 & 0.2 & 10.1 & 12.1 & 15.3 & 7.2 & 1.8 & 3.0 & 2.8 & 1.5 & 1.9 & 2.5 & 2.0 & 1.3 & 0.5 & 1.1 \\
\hline AMS5 & $01 / 04 / 2011$ & $1.5 \mathrm{E}-02$ & $5.6 \mathrm{E}-02$ & 6.0 & 9.3 & 17.2 & 10.1 & 2.3 & 3.9 & 3.6 & 1.9 & 2.7 & 2.8 & 1.6 & 0.6 & 0.2 & 0.5 \\
\hline AMS5 & $01 / 05 / 2011$ & $8.3 \mathrm{E}-02$ & 0.2 & 2.1 & 4.9 & 14.6 & 14.4 & 5.0 & 10.0 & 9.9 & 7.3 & 10.2 & 19.0 & 20.3 & 42.1 & 1.3 & 3.1 \\
\hline AMS5 & $02 / 06 / 2011$ & $3.8 \mathrm{E}-02$ & 0.1 & 1.4 & 4.6 & 18.5 & 22.3 & 4.4 & 9.2 & 8.2 & 4.8 & 5.4 & 6.7 & 4.7 & 6.3 & 0.5 & 1.2 \\
\hline AMS5 & $19 / 07 / 2011$ & $2.1 \mathrm{E}-03$ & $1.9 \mathrm{E}-02$ & 0 & 3.2 & 12.3 & 15.5 & 2.6 & 7.1 & 6.7 & 4.4 & 2.5 & 3.1 & 2.0 & 1.0 & 0.1 & 0.3 \\
\hline AMS5 & $02 / 08 / 2011$ & $2.6 \mathrm{E}-03$ & $1.5 \mathrm{E}-02$ & 1.5 & 4.9 & 11.3 & 12.3 & 2.6 & 6.8 & 6.3 & 3.6 & 2.2 & 2.5 & 1.6 & 0.7 & $8.8 \mathrm{E}-02$ & 0.2 \\
\hline AMS5 & $01 / 09 / 2011$ & $5.8 \mathrm{E}-03$ & $4.2 \mathrm{E}-02$ & 1.1 & 3.0 & 10.5 & 11.7 & 1.6 & 4.1 & 3.6 & 2.3 & 1.4 & 1.8 & 1.3 & 0.6 & $8.5 \mathrm{E}-02$ & 0.2 \\
\hline AMS5 & $30 / 09 / 2011$ & $6.3 \mathrm{E}-03$ & $5.0 \mathrm{E}-02$ & 0.8 & 2.7 & 8.6 & 9.1 & 1.5 & 3.3 & 3.0 & 1.8 & 1.5 & 1.9 & 1.5 & 0.7 & 0.1 & 0.3 \\
\hline AMS5 & $01 / 11 / 2011$ & $7.2 \mathrm{E}-02$ & 0.2 & 5.7 & 9.8 & 14.7 & 7.5 & 1.4 & 2.5 & 2.5 & 1.6 & 1.8 & 2.7 & 2.0 & 1.3 & 0.5 & 1.2 \\
\hline AMS5 & $30 / 11 / 2011$ & $9.4 \mathrm{E}-03$ & $5.9 \mathrm{E}-02$ & 2.2 & 6.8 & 11.8 & 5.9 & 1.5 & 2.5 & 2.2 & 1.3 & 1.3 & 1.2 & 0.7 & 0.5 & 0.1 & 0.3 \\
\hline AMS5 & $30 / 12 / 2011$ & $2.6 \mathrm{E}-02$ & 0.1 & 16.6 & 17.1 & 18.5 & 8.1 & 1.9 & 2.9 & 2.7 & 1.8 & 2.1 & 2.8 & 2.2 & 2.1 & 0.4 & 0.9 \\
\hline AMS5 & $31 / 01 / 2012$ & 0.2 & 0.5 & 24.5 & 24.5 & 22.9 & 9.0 & 3.0 & 4.2 & 5.1 & 2.7 & 5.2 & 6.4 & 3.8 & 3.5 & 1.6 & 3.8 \\
\hline AMS5 & 29/02/2012 & 0.2 & 0.4 & 7.4 & 14.7 & 27.7 & 13.2 & 2.5 & 4.3 & 3.7 & 1.8 & 3.3 & 4.2 & 2.5 & 1.1 & 1.1 & 2.9 \\
\hline AMS5 & $30 / 03 / 2012$ & $2.6 \mathrm{E}-02$ & $9.0 \mathrm{E}-02$ & 3.6 & 5.6 & 20.5 & 15.1 & 2.0 & 4.9 & 5.3 & 3.3 & 2.4 & 4.3 & 3.2 & 1.6 & 0.4 & 1.2 \\
\hline AMS5 & $30 / 04 / 2012$ & $2.4 \mathrm{E}-02$ & $7.8 \mathrm{E}-02$ & 1.4 & 2.5 & 14.5 & 14.5 & 2.5 & 6.6 & 5.6 & 3.6 & 2.5 & 3.9 & 2.6 & 1.1 & 0.3 & 0.8 \\
\hline AMS5 & $31 / 05 / 2012$ & $3.5 \mathrm{E}-02$ & 0.1 & 1.7 & 3.3 & 11.9 & 13.9 & 5.0 & 11.3 & 9.1 & 5.3 & 5.1 & 6.5 & 3.8 & 3.5 & 0.5 & 1.4 \\
\hline
\end{tabular}


Table S2: Monthly average air concentrations $\left(\mathrm{ng} / \mathrm{m}^{3}\right)$ based on 4-5 weekly air concentrations cont'd

\begin{tabular}{|c|c|c|c|c|c|c|c|c|c|c|c|c|c|c|c|c|c|}
\hline Site & Start date & DahAN & BghiPER & $\begin{array}{c}\text { C1 } \\
\text { NAP } \\
\end{array}$ & $\begin{array}{c}\text { C2 } \\
\text { NAP } \\
\end{array}$ & $\begin{array}{c}\text { C3 } \\
\text { NAP } \\
\end{array}$ & $\begin{array}{c}\text { C4 } \\
\text { NAP } \\
\end{array}$ & $\mathrm{C} 1 \mathrm{FL}$ & $\mathrm{C} 2 \mathrm{FL}$ & $\mathrm{C} 3 \mathrm{FL}$ & $\mathrm{C} 4 \mathrm{FL}$ & $\begin{array}{c}\text { C1 } \\
\text { PHE/AN }\end{array}$ & $\begin{array}{c}\mathrm{C} 2 \\
\text { PHE/AN }\end{array}$ & $\begin{array}{c}\text { C3 } \\
\text { PHE/AN }\end{array}$ & $\begin{array}{c}\mathrm{C} 4 \\
\text { PHE/AN }\end{array}$ & $\begin{array}{c}\text { C1 } \\
\text { FLT/PYR }\end{array}$ & $\begin{array}{c}\text { C2 } \\
\text { FLT/PYR }\end{array}$ \\
\hline AMS11 & $02 / 01 / 2011$ & 0.2 & 0.3 & 12.0 & 14.0 & 32.8 & 19.8 & 5.0 & 8.1 & 6.3 & 1.8 & 3.7 & 3.8 & 3.6 & 1.7 & 1.0 & 2.8 \\
\hline AMS11 & $31 / 01 / 2011$ & $5.9 \mathrm{E}-02$ & 0.2 & 6.2 & 10.5 & 15.4 & 8.4 & 2.5 & 4.1 & 3.4 & 1.5 & 2.5 & 2.4 & 2.0 & 1.4 & 0.6 & 1.1 \\
\hline AMS11 & 02/03/2011 & 7.6E-02 & 0.2 & 6.2 & 8.2 & 16.1 & 10.8 & 2.8 & 5.7 & 5.8 & 2.9 & 3.7 & 4.3 & 3.1 & 1.5 & 0.6 & 1.7 \\
\hline AMS11 & $01 / 04 / 2011$ & $6.7 \mathrm{E}-02$ & 0.1 & 5.8 & 13.9 & 18.9 & 10.0 & 1.9 & 3.8 & 2.9 & 1.3 & 1.4 & 1.6 & 1.0 & 0.3 & 0.5 & 1.3 \\
\hline AMS11 & $30 / 04 / 2011$ & 0.5 & 1.2 & 3.9 & 7.9 & 21.7 & 21.0 & 8.5 & 18.3 & 16.4 & 10.6 & 11.8 & 21.0 & 18.2 & 29.0 & 2.4 & 6.1 \\
\hline AMS11 & $02 / 06 / 2011$ & $6.9 \mathrm{E}-02$ & 0.2 & 3.3 & 3.8 & 15.2 & 16.9 & 5.1 & 11.4 & 10.3 & 6.4 & 4.7 & 6.1 & 4.5 & 6.0 & 0.6 & 1.4 \\
\hline AMS11 & $19 / 07 / 2011$ & $1.6 \mathrm{E}-02$ & $3.8 \mathrm{E}-02$ & 0 & 1.9 & 16.0 & 21.8 & 5.5 & 14.9 & 15.8 & 9.8 & 4.2 & 5.4 & 3.6 & 1.4 & 0.3 & 0.5 \\
\hline AMS11 & $02 / 08 / 2011$ & $1.5 \mathrm{E}-02$ & $2.1 \mathrm{E}-02$ & 2.4 & 5.9 & 19.2 & 20.7 & 5.4 & 13.9 & 12.2 & 6.8 & 3.4 & 4.1 & 2.5 & 0.8 & 0.2 & 0.4 \\
\hline AMS11 & 01/09/2011 & $2.2 \mathrm{E}-02$ & $3.6 \mathrm{E}-02$ & 0.5 & 3.1 & 17.3 & 19.9 & 4.6 & 10.5 & 8.9 & 4.7 & 3.5 & 3.7 & 1.9 & 0.7 & 0.3 & 0.3 \\
\hline AMS11 & $30 / 09 / 2011$ & 0.1 & 0.2 & 1.8 & 3.4 & 15.0 & 13.1 & 2.8 & 5.9 & 5.2 & 2.9 & 2.4 & 2.9 & 1.8 & 0.7 & 0.7 & 1.7 \\
\hline AMS11 & $01 / 11 / 2011$ & 0.6 & 1.0 & 5.1 & 9.7 & 16.8 & 9.0 & 2.7 & 5.0 & 6.7 & 3.5 & 5.3 & 7.6 & 5.1 & 3.0 & 2.6 & 6.9 \\
\hline AMS11 & $30 / 11 / 2011$ & 0.4 & 0.8 & 4.6 & 11.3 & 32.4 & 20.9 & 5.0 & 8.9 & 7.3 & 3.2 & 5.1 & 5.9 & 3.1 & 1.6 & 1.9 & 4.9 \\
\hline AMS11 & $20 / 12 / 2011$ & 0.3 & 0.5 & 11.2 & 9.9 & 17.1 & 11.0 & 3.1 & 4.5 & 4.1 & 1.7 & 3.2 & 3.4 & 1.8 & 1.2 & 1.1 & 2.7 \\
\hline AMS11 & $30 / 01 / 2012$ & 1.0 & 2.0 & 11.4 & 14.0 & 24.8 & 16.2 & 5.5 & 9.1 & 9.4 & 4.1 & 8.9 & 11.0 & 6.5 & 4.5 & 4.2 & 11.4 \\
\hline AMS11 & $29 / 02 / 2012$ & 0.1 & 0.3 & 5.7 & 8.6 & 27.7 & 17.6 & 3.7 & 7.2 & 6.0 & 2.9 & 3.3 & 4.0 & 2.2 & 0.9 & 0.7 & 2.0 \\
\hline AMS11 & $30 / 03 / 2012$ & 0.1 & 0.2 & 3.3 & 6.1 & 23.4 & 20.7 & 4.4 & 8.7 & 7.6 & 4.0 & 4.6 & 6.3 & 3.8 & 1.8 & 0.8 & 2.3 \\
\hline AMS11 & $30 / 04 / 2012$ & $5.2 \mathrm{E}-02$ & 0.1 & 1.2 & 2.3 & 15.8 & 22.3 & 5.1 & 11.4 & 9.2 & 5.4 & 4.2 & 5.6 & 3.1 & 1.1 & 0.4 & 1.0 \\
\hline AMS11 & $31 / 05 / 2012$ & 7.3E-02 & 0.2 & 0.9 & 2.5 & 9.9 & 10.3 & 4.6 & 10.0 & 8.3 & 4.9 & 4.2 & 5.7 & 3.4 & 4.1 & 0.6 & 1.6 \\
\hline
\end{tabular}


Table S2: Monthly average air concentrations $\left(\mathrm{ng} / \mathrm{m}^{3}\right)$ based on 4-5 weekly air concentrations cont'd

\begin{tabular}{|c|c|c|c|c|c|c|c|c|c|c|c|c|c|c|c|c|c|}
\hline Site & Start date & DahAN & BghiPER & C1 NAP & $\begin{array}{c}\mathrm{C} 2 \\
\mathrm{NAP} \\
\end{array}$ & $\begin{array}{c}\text { C3 } \\
\text { NAP } \\
\end{array}$ & $\begin{array}{c}\text { C4 } \\
\text { NAP } \\
\end{array}$ & $\mathrm{C} 1 \mathrm{FL}$ & $\mathrm{C} 2 \mathrm{FL}$ & $\mathrm{C} 3 \mathrm{FL}$ & C4 FL & $\begin{array}{c}\text { C1 } \\
\text { PHE/AN }\end{array}$ & $\begin{array}{c}\mathrm{C} 2 \\
\text { PHE/AN }\end{array}$ & $\begin{array}{c}\text { C3 } \\
\text { PHE/AN }\end{array}$ & $\begin{array}{c}\mathrm{C} 4 \\
\text { PHE/AN }\end{array}$ & $\begin{array}{c}\text { C1 } \\
\text { FLT/PYR }\end{array}$ & $\begin{array}{c}\text { C2 } \\
\text { FLT/PYR }\end{array}$ \\
\hline AMS13 & $24 / 01 / 2011$ & $9.8 \mathrm{E}-03$ & $5.5 \mathrm{E}-02$ & 5.4 & 17.8 & 25.1 & 12.5 & 2.5 & 4.2 & 2.9 & 1.3 & 2.0 & 1.5 & 1.2 & 1.3 & 0.2 & 0.3 \\
\hline AMS13 & $31 / 01 / 2011$ & $6.1 \mathrm{E}-03$ & $5.2 \mathrm{E}-02$ & 5.7 & 7.4 & 7.0 & 4.0 & 1.1 & 1.8 & 1.3 & 0.4 & 0.8 & 0.7 & 0.6 & 0.7 & 0.1 & 0.3 \\
\hline AMS13 & 02/03/2011 & $1.3 \mathrm{E}-02$ & 5.7E-02 & 2.2 & 4.7 & 9.7 & 6.0 & 1.2 & 2.2 & 2.1 & 1.0 & 1.6 & 2.0 & 2.0 & 1.5 & 0.2 & 0.4 \\
\hline AMS13 & $01 / 04 / 2011$ & NA & 3.7E-03 & 3.3 & 11.3 & 22.3 & 14.5 & 2.1 & 3.6 & 2.9 & 1.3 & 1.7 & 2.0 & 1.4 & 0.6 & $6.6 \mathrm{E}-02$ & 0.2 \\
\hline AMS13 & $30 / 04 / 2011$ & 0.1 & 0.4 & 2.7 & 6.7 & 25.7 & 21.8 & 8.3 & 13.8 & 11.3 & 8.2 & 11.4 & 22.7 & 23.1 & 64.0 & 1.5 & 3.8 \\
\hline AMS13 & $02 / 06 / 2011$ & $1.7 \mathrm{E}-02$ & $9.2 \mathrm{E}-02$ & 0.9 & 2.4 & 7.5 & 8.6 & 3.7 & 6.8 & 5.4 & 3.6 & 3.5 & 5.0 & 5.1 & 9.9 & 0.4 & 1.3 \\
\hline AMS13 & $19 / 07 / 2011$ & NA & NA & NA & NA & 1.5 & 2.1 & 0.8 & 2.0 & 1.7 & 1.1 & 0.5 & 0.6 & 0.3 & 0.3 & 4.0E-03 & NA \\
\hline AMS13 & $02 / 08 / 2011$ & NA & NA & NA & NA & 2.1 & 2.4 & 1.0 & 2.3 & 2.0 & 1.0 & 0.8 & 1.0 & 0.5 & 0.3 & 7.5E-03 & NA \\
\hline AMS13 & $01 / 09 / 2011$ & $2.6 \mathrm{E}-03$ & $2.5 \mathrm{E}-02$ & NA & 0.9 & 1.5 & 1.7 & 0.6 & 1.4 & 1.1 & 0.6 & 0.2 & 0.3 & 0.2 & 0.2 & $6.7 \mathrm{E}-03$ & $3.9 \mathrm{E}-03$ \\
\hline AMS13 & $30 / 09 / 2011$ & 3.7E-03 & $2.0 \mathrm{E}-02$ & $5.8 \mathrm{E}-02$ & 0.7 & 4.9 & 5.5 & 1.0 & 2.1 & 1.5 & 0.8 & 0.5 & 0.7 & 0.4 & 0.3 & $4.0 \mathrm{E}-02$ & $9.0 \mathrm{E}-02$ \\
\hline AMS13 & $01 / 11 / 2011$ & $4.3 \mathrm{E}-03$ & $3.3 \mathrm{E}-02$ & 2.0 & 4.1 & 7.0 & 4.2 & 0.8 & 1.5 & 1.4 & 0.8 & 0.6 & 1.0 & 0.9 & 0.7 & 0.1 & 0.3 \\
\hline AMS13 & $30 / 11 / 2011$ & $1.0 \mathrm{E}-02$ & 4.4E-02 & 3.1 & 6.2 & 9.4 & 4.6 & 1.0 & 1.5 & 1.0 & 0.5 & 0.6 & 0.5 & 0.3 & 0.3 & $9.9 \mathrm{E}-02$ & 0.2 \\
\hline AMS13 & $30 / 12 / 2011$ & $1.2 \mathrm{E}-02$ & $5.5 \mathrm{E}-02$ & 6.2 & 6.0 & 6.3 & 2.8 & 0.7 & 1.3 & 0.8 & 0.5 & 0.8 & 1.0 & 0.8 & 1.0 & 0.1 & 0.3 \\
\hline AMS13 & $31 / 01 / 2012$ & $6.4 \mathrm{E}-02$ & 0.2 & 7.7 & 11.5 & 14.0 & 6.6 & 1.6 & 2.6 & 2.3 & 1.2 & 1.7 & 2.5 & 1.7 & 1.5 & 0.4 & 1.2 \\
\hline AMS13 & $29 / 02 / 2012$ & $8.1 \mathrm{E}-03$ & $2.2 \mathrm{E}-02$ & 1.6 & 3.8 & 17.4 & 10.3 & 2.2 & 3.7 & 2.4 & 1.0 & 1.2 & 1.2 & 0.6 & 0.3 & 7.3E-02 & 0.4 \\
\hline AMS13 & $30 / 03 / 2012$ & $5.3 \mathrm{E}-03$ & $1.2 \mathrm{E}-02$ & 2.7 & 4.2 & 10.3 & 6.3 & 1.4 & 2.7 & 1.8 & 0.9 & 1.0 & 1.3 & 0.7 & 0.5 & $8.2 \mathrm{E}-02$ & 0.3 \\
\hline AMS13 & $30 / 04 / 2012$ & $1.7 \mathrm{E}-02$ & 4.0E-02 & 0.6 & 0.9 & 2.2 & 2.4 & 1.2 & 2.6 & 1.7 & 0.9 & 1.1 & 1.1 & 0.6 & 0.3 & 0.1 & 0.3 \\
\hline AMS13 & $31 / 05 / 2012$ & $5.3 \mathrm{E}-03$ & $2.1 \mathrm{E}-02$ & 0.2 & 0.9 & 6.7 & 6.9 & 2.7 & 6.4 & 4.3 & 2.6 & 2.0 & 2.4 & 1.3 & 2.1 & $8.2 \mathrm{E}-02$ & 0.3 \\
\hline
\end{tabular}


Table S2: Monthly average air concentrations $\left(\mathrm{ng} / \mathrm{m}^{3}\right)$ based on 4-5 weekly air concentrations cont'd

\begin{tabular}{|l|l|l|l|l|l|l|l|l|l|c|c|c|}
\hline Site & Start date & $\begin{array}{c}\text { C3 } \\
\text { FLT/PYR }\end{array}$ & $\begin{array}{c}\text { C4 } \\
\text { FLT/PYR }\end{array}$ & C1 BTC & C2 BTC & C3 BTC & C4 BTC & DBT & $\begin{array}{c}\text { C1 } \\
\text { DBT }\end{array}$ & $\begin{array}{c}\text { C2 } \\
\text { DBT }\end{array}$ & $\begin{array}{c}\text { C3 } \\
\text { DBT }\end{array}$ & $\begin{array}{c}\text { C4 } \\
\text { DBT }\end{array}$ \\
\hline AMS5 & $02 / 01 / 2011$ & - & - & - & - & - & - & - & - & - & - & - \\
\hline AMS5 & $31 / 01 / 2011$ & 1.5 & 0.9 & 0.8 & 0.6 & 0.7 & 0.5 & 0.1 & 1.3 & 3.9 & 5.6 & 7.5 \\
\hline AMS5 & $02 / 03 / 2011$ & 1.4 & 0.7 & 1.1 & 0.8 & 0.6 & 0.4 & 0.4 & 0.7 & 0.2 & NA & NA \\
\hline AMS5 & $01 / 04 / 2011$ & 0.7 & 0.4 & 0.4 & 0.3 & 0.3 & 0.2 & NA & NA & NA & NA & NA \\
\hline AMS5 & $01 / 05 / 2011$ & 3.8 & 1.9 & 2.0 & 2.2 & 2.3 & 1.4 & 2.6 & 8.8 & 11.1 & 5.9 & 4.3 \\
\hline AMS5 & $02 / 06 / 2011$ & 1.4 & 0.8 & 0.9 & 0.9 & 0.9 & 0.7 & 1.0 & 5.6 & 7.5 & 4.6 & 3.4 \\
\hline AMS5 & $19 / 07 / 2011$ & 0.4 & 0.2 & 0.1 & 0.1 & 0.2 & 0.2 & 0.4 & 2.7 & 3.9 & 2.6 & 2.0 \\
\hline AMS5 & $02 / 08 / 2011$ & 0.3 & 0.2 & 0.1 & 0.1 & 0.2 & 0.1 & 0.4 & 2.3 & 3.2 & 2.0 & 1.7 \\
\hline AMS5 & $01 / 09 / 2011$ & 0.3 & 0.2 & 0.2 & 0.2 & 0.2 & 0.2 & 0.3 & 1.4 & 2.1 & 1.5 & 1.2 \\
\hline AMS5 & $30 / 09 / 2011$ & 0.4 & 0.3 & 0.2 & 0.2 & 0.2 & 0.2 & 0.4 & 1.5 & 2.2 & 1.5 & 1.3 \\
\hline AMS5 & $01 / 11 / 2011$ & 1.8 & 1.2 & 1.3 & 0.9 & 0.8 & 0.6 & 0.3 & 1.6 & 2.6 & 2.1 & 2.1 \\
\hline AMS5 & $30 / 11 / 2011$ & 0.3 & 0.2 & 0.2 & 0.1 & 0.1 & 0.1 & 0.2 & 0.8 & 0.8 & 0.3 & 0.1 \\
\hline AMS5 & $30 / 12 / 2011$ & 1.1 & 0.7 & 0.6 & 0.4 & 0.4 & 0.4 & 0.3 & 1.3 & 1.7 & 2.1 & 2.3 \\
\hline AMS5 & $31 / 01 / 2012$ & 4.5 & 3.0 & 4.3 & 2.9 & 2.4 & 1.6 & 1.0 & 3.3 & 4.4 & 3.0 & 2.6 \\
\hline AMS5 & $29 / 02 / 2012$ & 3.8 & 2.6 & 3.4 & 2.5 & 2.2 & 1.4 & 0.8 & 3.3 & 4.5 & 3.0 & 2.3 \\
\hline AMS5 & $30 / 03 / 2012$ & 1.9 & 1.3 & 0.8 & 0.8 & 1.0 & 0.8 & 0.2 & 1.7 & 3.7 & 3.6 & 3.7 \\
\hline AMS5 & $30 / 04 / 2012$ & 1.2 & 0.8 & 0.7 & 0.6 & 0.7 & 0.5 & 0.2 & 2.1 & 4.1 & 3.4 & 3.1 \\
\hline AMS5 & $31 / 05 / 2012$ & 1.8 & 1.2 & 1.1 & 0.9 & 1.0 & 0.8 & 1.3 & 5.9 & 7.8 & 5.0 & 4.1 \\
\hline
\end{tabular}


Table S2: Monthly average air concentrations $\left(\mathrm{ng} / \mathrm{m}^{3}\right)$ based on 4-5 weekly air concentrations cont'd

\begin{tabular}{|c|c|c|c|c|c|c|c|c|c|c|c|c|}
\hline Site & Start date & $\begin{array}{c}\text { C3 } \\
\text { FLT/PYR }\end{array}$ & $\begin{array}{c}\text { C4 } \\
\text { FLT/PYR }\end{array}$ & C1 BTC & C2 BTC & C3 BTC & C4 BTC & DBT & $\begin{array}{c}\mathrm{C} 1 \\
\mathrm{DBT}\end{array}$ & $\begin{array}{c}\mathrm{C} 2 \\
\mathrm{DBT}\end{array}$ & $\begin{array}{c}\mathrm{C} 3 \\
\mathrm{DBT}\end{array}$ & $\begin{array}{c}\text { C4 } \\
\text { DBT }\end{array}$ \\
\hline AMS11 & 02/01/2011 & 3.5 & 2.3 & 3.3 & 1.9 & 1.6 & 1.1 & 1.5 & 5.0 & 5.9 & 3.7 & 2.9 \\
\hline AMS11 & $31 / 01 / 2011$ & 1.4 & 0.8 & 1.2 & 0.7 & 0.6 & 0.4 & 1.1 & 3.7 & 4.5 & 2.6 & 2.2 \\
\hline AMS11 & $02 / 03 / 2011$ & 2.6 & 1.4 & 1.8 & 1.2 & 1.1 & 0.8 & 1.6 & 9.8 & 14.3 & 11.3 & 10.0 \\
\hline AMS11 & $01 / 04 / 2011$ & 1.7 & 1.2 & 1.4 & 1.0 & 0.9 & 0.7 & NA & NA & NA & NA & NA \\
\hline AMS11 & $30 / 04 / 2011$ & 7.6 & 5.2 & 7.7 & 4.6 & 4.8 & 2.8 & 1.7 & 8.0 & 12.7 & 9.2 & 9.5 \\
\hline AMS11 & $02 / 06 / 2011$ & 1.8 & 1.1 & 1.3 & 1.2 & 1.1 & 0.8 & 0.7 & 4.1 & 6.6 & 4.5 & 3.4 \\
\hline AMS11 & $19 / 07 / 2011$ & 0.4 & 0.3 & 0.7 & 0.2 & 0.2 & 0.2 & 0.6 & 4.2 & 6.9 & 5.1 & 4.2 \\
\hline AMS11 & $02 / 08 / 2011$ & 1.1 & 0.2 & 0.3 & 0.3 & 0.2 & 0.2 & 0.7 & 3.6 & 5.5 & 3.8 & 3.0 \\
\hline AMS11 & $01 / 09 / 2011$ & 0.6 & 0.3 & 0.4 & 0.2 & 0.2 & 0.1 & 0.8 & 4.0 & 5.4 & 3.2 & 2.3 \\
\hline AMS11 & $30 / 09 / 2011$ & 2.2 & 1.6 & 1.8 & 1.2 & 1.1 & 0.7 & 0.6 & 2.8 & 4.3 & 3.1 & 2.3 \\
\hline AMS11 & $01 / 11 / 2011$ & 9.3 & 6.6 & 7.7 & 5.4 & 4.8 & 3.1 & 1.4 & 6.1 & 10.1 & 8.2 & 7.4 \\
\hline AMS11 & $30 / 11 / 2011$ & 6.0 & 4.2 & 5.8 & 3.8 & 2.8 & 1.8 & 1.1 & 5.9 & 8.3 & 5.6 & 4.1 \\
\hline AMS11 & $20 / 12 / 2011$ & 3.0 & 2.1 & 3.4 & 2.0 & 1.5 & 1.0 & 0.9 & 3.7 & 4.9 & 3.1 & 2.3 \\
\hline AMS11 & $30 / 01 / 2012$ & 13.7 & 10.1 & 14.9 & 9.4 & 8.2 & 5.3 & 2.3 & 10.6 & 15.4 & 10.8 & 8.5 \\
\hline AMS11 & $29 / 02 / 2012$ & 2.7 & 1.9 & 2.2 & 1.5 & 1.5 & 1.0 & 0.7 & 3.7 & 5.5 & 3.8 & 3.0 \\
\hline AMS11 & $30 / 03 / 2012$ & 3.3 & 2.4 & 2.4 & 2.0 & 1.9 & 1.3 & 1.0 & 5.3 & 8.3 & 6.3 & 5.4 \\
\hline AMS11 & $30 / 04 / 2012$ & 1.3 & 0.9 & 1.0 & 0.7 & 0.8 & 0.6 & 0.7 & 4.8 & 7.4 & 5.3 & 4.1 \\
\hline AMS11 & $31 / 05 / 2012$ & 2.1 & 1.4 & 1.6 & 1.3 & 1.2 & 0.9 & 0.8 & 4.3 & 6.5 & 4.9 & 4.1 \\
\hline
\end{tabular}


Table S2: Monthly average air concentrations $\left(\mathrm{ng} / \mathrm{m}^{3}\right)$ based on 4-5 weekly air concentrations cont'd

\begin{tabular}{|c|c|c|c|c|c|c|c|c|c|c|c|c|}
\hline Site & Start date & $\begin{array}{c}\text { C3 } \\
\text { FLT/PYR }\end{array}$ & $\begin{array}{c}\text { C4 } \\
\text { FLT/PYR }\end{array}$ & C1 BTC & C2 BTC & C3 BTC & C4 BTC & DBT & C1 DBT & C2 DBT & C3 DBT & C4 DBT \\
\hline AMS13 & $24 / 01 / 2011$ & 0.3 & 0.1 & 0.3 & 0.2 & 8.7E-02 & 0.1 & 0.6 & 2.2 & 2.6 & 1.2 & 0.8 \\
\hline AMS13 & $31 / 01 / 2011$ & 0.3 & 0.1 & 0.2 & 0.1 & 0.1 & 0.1 & NA & NA & NA & NA & NA \\
\hline AMS13 & $02 / 03 / 2011$ & 0.7 & 0.4 & 0.3 & 0.3 & 0.3 & 0.3 & NA & NA & NA & NA & NA \\
\hline AMS13 & $01 / 04 / 2011$ & 0.5 & 0.3 & 0.1 & 0.1 & 0.2 & 0.2 & NA & NA & NA & NA & NA \\
\hline AMS13 & $30 / 04 / 2011$ & 5.3 & 2.5 & 3.2 & 4.2 & 4.6 & 2.5 & 0.4 & 2.4 & 6.3 & 4.3 & 3.3 \\
\hline AMS13 & $02 / 06 / 2011$ & 2.0 & 1.0 & 0.8 & 1.2 & 1.1 & 0.7 & 0.2 & 1.3 & 2.7 & 1.7 & 1.4 \\
\hline AMS13 & $19 / 07 / 2011$ & NA & NA & NA & NA & $2.0 \mathrm{E}-02$ & $3.2 \mathrm{E}-02$ & $5.9 \mathrm{E}-02$ & 0.5 & 0.8 & 0.5 & 0.4 \\
\hline AMS13 & 02/08/2011 & NA & NA & NA & NA & NA & $3.2 \mathrm{E}-02$ & $6.6 \mathrm{E}-02$ & 0.4 & 0.8 & 0.4 & 0.5 \\
\hline AMS13 & 01/09/2011 & $4.0 \mathrm{E}-02$ & $1.4 \mathrm{E}-02$ & 1.6E-02 & 5.3E-02 & 3.7E-02 & $6.5 \mathrm{E}-02$ & $4.4 \mathrm{E}-02$ & 0.2 & 0.3 & 0.2 & 0.2 \\
\hline AMS13 & $30 / 09 / 2011$ & 0.2 & 0.1 & 9.3E-02 & $5.8 \mathrm{E}-02$ & $6.9 \mathrm{E}-02$ & 0.1 & 8.0E-02 & 0.4 & 0.8 & 0.6 & 0.6 \\
\hline AMS13 & $01 / 11 / 2011$ & 0.5 & 0.3 & 0.1 & 0.1 & 0.2 & 0.2 & $5.3 \mathrm{E}-02$ & 0.4 & 0.9 & 1.0 & 1.2 \\
\hline AMS13 & $30 / 11 / 2011$ & 0.2 & 0.1 & 0.2 & 0.1 & 1.0E-01 & $8.4 \mathrm{E}-02$ & 0.1 & 0.6 & 0.8 & 0.4 & 0.2 \\
\hline AMS13 & $30 / 12 / 2011$ & 0.3 & 0.2 & 0.2 & 0.2 & 0.1 & 0.1 & 0.1 & 0.4 & 0.6 & 0.4 & 0.4 \\
\hline AMS13 & $31 / 01 / 2012$ & 1.6 & 1.0 & 1.2 & 0.9 & 0.8 & 0.7 & 0.4 & 1.6 & 2.4 & 1.9 & 2.0 \\
\hline AMS13 & 29/02/2012 & 0.3 & 0.3 & 0.3 & 0.3 & 0.4 & 0.2 & 0.2 & 1.5 & 2.3 & 1.6 & 1.5 \\
\hline AMS13 & $30 / 03 / 2012$ & 0.4 & 0.3 & 0.2 & 0.2 & 0.2 & 0.2 & 0.2 & 1.1 & 1.7 & 1.2 & 1.1 \\
\hline AMS13 & $30 / 04 / 2012$ & 0.4 & 0.2 & 0.3 & 0.3 & 0.2 & 0.2 & 0.4 & 1.6 & 2.0 & 1.0 & 0.8 \\
\hline AMS13 & $31 / 05 / 2012$ & 0.5 & 0.1 & 0.2 & 0.3 & 0.3 & 0.2 & 0.1 & 1.3 & 2.6 & 1.7 & 1.3 \\
\hline
\end{tabular}


Table S3: Descriptive statistics of the total scavenging ratios $\left(\mathrm{W}_{\mathrm{t}}\right)$ for snow and rain at the oil sands sites. $(\mathrm{G})$ and $(\mathrm{P})$ denote predominantly gas-phase and particulate-phase PACs, respectively

\begin{tabular}{|c|c|c|c|c|c|c|c|c|c|c|}
\hline \multirow[t]{2}{*}{ PACs } & \multicolumn{2}{|c|}{ \# of samples } & \multicolumn{2}{|c|}{ Median } & \multicolumn{2}{|c|}{ Mean } & \multicolumn{2}{|c|}{ Min } & \multicolumn{2}{|c|}{ Max } \\
\hline & Snow & Rain & Snow & Rain & Snow & Rain & Snow & Rain & Snow & Rain \\
\hline Naphthalene (G) & 19 & 15 & $4.7 \times 10^{4}$ & $3.6 \times 10^{4}$ & $4.6 \times 10^{5}$ & $5.3 \times 10^{5}$ & $1.7 \times 10^{3}$ & $1.2 \times 10^{2}$ & $2.5 \times 10^{6}$ & $4.7 \times 10^{6}$ \\
\hline Acenaphthylene (G) & 19 & 27 & $6.8 \times 10^{3}$ & $3.5 \times 10^{2}$ & $9.9 \times 10^{3}$ & $7.8 \times 10^{3}$ & $7.2 \times 10^{2}$ & 33.6 & $2.9 \times 10^{4}$ & $5.3 \times 10^{4}$ \\
\hline Acenaphthene (G) & 19 & 28 & $6.1 \times 10^{3}$ & $3.5 \times 10^{3}$ & $1.4 \times 10^{4}$ & $9.1 \times 10^{3}$ & $4.8 \times 10^{2}$ & $1.9 \times 10^{3}$ & $8.2 \times 10^{4}$ & $8.9 \times 10^{4}$ \\
\hline Fluorene $(G)$ & 19 & 28 & $3.2 \times 10^{4}$ & $5.8 \times 10^{3}$ & $7.8 \times 10^{4}$ & $1.0 \times 10^{4}$ & $3.2 \times 10^{3}$ & $1.5 \times 10^{3}$ & $5.5 \times 10^{5}$ & $1.1 \times 10^{5}$ \\
\hline Phenanthrene (G) & 19 & 28 & $1.1 \times 10^{5}$ & $1.5 \times 10^{4}$ & $2.5 \times 10^{5}$ & $2.2 \times 10^{4}$ & $1.6 \times 10^{4}$ & $3.2 \times 10^{3}$ & $9.1 \times 10^{5}$ & $1.1 \times 10^{5}$ \\
\hline Anthracene (G) & 18 & 28 & $4.1 \times 10^{5}$ & $3.5 \times 10^{4}$ & $1.4 \times 10^{6}$ & $8.5 \times 10^{4}$ & $5.1 \times 10^{4}$ & $1.7 \times 10^{3}$ & $7.8 \times 10^{6}$ & $5.5 \times 10^{5}$ \\
\hline Fluoranthene & 18 & 27 & $2.5 \times 10^{5}$ & $3.8 \times 10^{4}$ & $4.5 \times 10^{5}$ & $6.3 \times 10^{4}$ & $7.1 \times 10^{4}$ & $8.4 \times 10^{3}$ & $1.6 \times 10^{6}$ & $2.6 \times 10^{5}$ \\
\hline Pyrene & 17 & 24 & $3.4 \times 10^{5}$ & $7.1 \times 10^{4}$ & $1.1 \times 10^{6}$ & $1.2 \times 10^{5}$ & $1.1 \times 10^{5}$ & $1.8 \times 10^{4}$ & $4.7 \times 10^{6}$ & $5.8 \times 10^{5}$ \\
\hline Retene & 18 & 27 & $6.6 \times 10^{4}$ & $2.1 \times 10^{4}$ & $1.2 \times 10^{5}$ & $4.5 \times 10^{4}$ & $6.7 \times 10^{4}$ & 17.0 & $5.8 \times 10^{5}$ & $2.0 \times 10^{5}$ \\
\hline Benz $[a]$ anthracene $(\mathrm{P})$ & 17 & 16 & $8.2 \times 10^{5}$ & $2.1 \times 10^{5}$ & $2.9 \times 10^{6}$ & $2.8 \times 10^{5}$ & $2.5 \times 10^{5}$ & $2.2 \times 10^{4}$ & $1.2 \times 10^{7}$ & $9.8 \times 10^{5}$ \\
\hline Chrysene (P) & 17 & 20 & $6.0 \times 10^{5}$ & $1.7 \times 10^{5}$ & $2.3 \times 10^{6}$ & $2.8 \times 10^{5}$ & $1.9 \times 10^{5}$ & $2.0 \times 10^{4}$ & $8.4 \times 10^{6}$ & $1.3 \times 10^{6}$ \\
\hline Benzo[b]fluoranthene (P) & 18 & 21 & $5.3 \times 10^{5}$ & $1.2 \times 10^{5}$ & $1.6 \times 10^{6}$ & $1.5 \times 10^{5}$ & $1.2 \times 10^{5}$ & $1.3 \times 10^{4}$ & $7.3 \times 10^{6}$ & $6.4 \times 10^{5}$ \\
\hline Benzo[k]fluoranthene $(\mathrm{P})$ & 19 & 22 & $5.1 \times 10^{5}$ & $4.8 \times 10^{4}$ & $1.5 \times 10^{6}$ & $1.3 \times 10^{5}$ & $9.3 \times 10^{4}$ & $3.9 \times 10^{3}$ & $6.0 \times 10^{6}$ & $9.0 \times 10^{5}$ \\
\hline Benzo[a]pyrene $(\mathrm{P})$ & 18 & 21 & $9.8 \times 10^{5}$ & $1.9 \times 10^{5}$ & $4.3 \times 10^{6}$ & $3.8 \times 10^{5}$ & $1.9 \times 10^{5}$ & $2.3 \times 10^{4}$ & $1.9 \times 10^{7}$ & $1.9 \times 10^{6}$ \\
\hline Perylene & 16 & 20 & $6.4 \times 10^{5}$ & $2.1 \times 10^{5}$ & $2.7 \times 10^{6}$ & $3.8 \times 10^{5}$ & $1.8 \times 10^{5}$ & $3.0 \times 10^{4}$ & $1.3 \times 10^{7}$ & $1.5 \times 10^{6}$ \\
\hline Indeno $[1,2,3-c d]$ pyrene $(\mathrm{P})$ & 17 & 21 & $5.3 \times 10^{5}$ & $9.1 \times 10^{4}$ & $1.8 \times 10^{6}$ & $1.7 \times 10^{5}$ & $1.2 \times 10^{5}$ & $1.0 \times 10^{4}$ & $6.8 \times 10^{6}$ & $8.0 \times 10^{5}$ \\
\hline Dibenz $[a, h]$ anthracene $(\mathrm{P})$ & 18 & 20 & $1.1 \times 10^{6}$ & $1.9 \times 10^{5}$ & $3.5 \times 10^{6}$ & $3.7 \times 10^{5}$ & $2.2 \times 10^{5}$ & $1.1 \times 10^{4}$ & $1.3 \times 10^{7}$ & $1.7 \times 10^{6}$ \\
\hline Benzo[ghi]perylene (P) & 17 & 20 & $5.7 \times 10^{5}$ & $1.5 \times 10^{5}$ & $2.6 \times 10^{6}$ & $2.2 \times 10^{5}$ & $1.6 \times 10^{5}$ & $2.1 \times 10^{4}$ & $1.0 \times 10^{7}$ & $9.1 \times 10^{5}$ \\
\hline C1 Naphthalenes (G) & 19 & 16 & $1.6 \times 10^{4}$ & $6.2 \times 10^{3}$ & $2.2 \times 10^{4}$ & $1.9 \times 10^{4}$ & $1.3 \times 10^{3}$ & $9.5 \times 10^{2}$ & $6.7 \times 10^{4}$ & $1.8 \times 10^{5}$ \\
\hline C2 Naphthalenes (G) & 19 & 23 & $1.8 \times 10^{4}$ & $5.0 \times 10^{3}$ & $1.9 \times 10^{4}$ & $1.0 \times 10^{4}$ & $7.2 \times 10^{2}$ & $7.5 \times 10^{2}$ & $4.9 \times 10^{4}$ & $7.4 \times 10^{4}$ \\
\hline C3 Naphthalenes (G) & 19 & 28 & $1.1 \times 10^{4}$ & $2.9 \times 10^{3}$ & $1.3 \times 10^{4}$ & $4.8 \times 10^{3}$ & $4.1 \times 10^{2}$ & $9.7 \times 10^{2}$ & $3.8 \times 10^{4}$ & $2.9 \times 10^{4}$ \\
\hline C4 Naphthalenes (G) & 19 & 28 & $1.2 \times 10^{4}$ & $3.1 \times 10^{3}$ & $1.2 \times 10^{4}$ & $4.5 \times 10^{3}$ & $1.1 \times 10^{3}$ & $7.2 \times 10^{2}$ & $3.8 \times 10^{4}$ & $1.7 \times 10^{4}$ \\
\hline C1 Fluorenes (G) & 19 & 28 & $5.2 \times 10^{4}$ & $4.3 \times 10^{3}$ & $5.8 \times 10^{4}$ & $8.5 \times 10^{3}$ & $6.5 \times 10^{3}$ & $1.2 \times 10^{3}$ & $1.5 \times 10^{5}$ & $4.3 \times 10^{4}$ \\
\hline C2 Fluorenes (G) & 19 & 28 & $8.2 \times 10^{4}$ & $6.6 \times 10^{3}$ & $9.1 \times 10^{4}$ & $1.4 \times 10^{4}$ & $2.1 \times 10^{4}$ & $2.3 \times 10^{3}$ & $2.3 \times 10^{5}$ & $7.4 \times 10^{4}$ \\
\hline C3 Fluorenes (G) & 19 & 28 & $1.5 \times 10^{5}$ & $1.6 \times 10^{4}$ & $2.3 \times 10^{5}$ & $3.6 \times 10^{4}$ & $4.0 \times 10^{4}$ & $4.6 \times 10^{3}$ & $6.4 \times 10^{5}$ & $2.5 \times 10^{5}$ \\
\hline C4 Fluorenes (G) & 19 & 28 & $1.5 \times 10^{5}$ & $2.1 \times 10^{4}$ & $2.2 \times 10^{5}$ & $3.8 \times 10^{4}$ & $3.0 \times 10^{4}$ & $5.6 \times 10^{3}$ & $7.8 \times 10^{5}$ & $2.4 \times 10^{5}$ \\
\hline C1 Phenanthrene/Anthracene (G) & 19 & 27 & $2.2 \times 10^{5}$ & $2.5 \times 10^{4}$ & $3.8 \times 10^{5}$ & $5.4 \times 10^{4}$ & $4.5 \times 10^{4}$ & $5.9 \times 10^{3}$ & $9.9 \times 10^{5}$ & $3.6 \times 10^{5}$ \\
\hline C2 Phenanthrene/Anthracene & 19 & 25 & $2.5 \times 10^{5}$ & $4.3 \times 10^{4}$ & $4.6 \times 10^{5}$ & $8.3 \times 10^{4}$ & $9.8 \times 10^{4}$ & $9.9 \times 10^{3}$ & $1.4 \times 10^{6}$ & $6.5 \times 10^{5}$ \\
\hline C3 Phenanthrene/Anthracene & 19 & 23 & $2.9 \times 10^{5}$ & $6.6 \times 10^{4}$ & $4.6 \times 10^{5}$ & $8.9 \times 10^{4}$ & $8.6 \times 10^{4}$ & $9.9 \times 10^{3}$ & $1.2 \times 10^{6}$ & $2.5 \times 10^{5}$ \\
\hline C4 Phenanthrene/Anthracene & 18 & 28 & $3.1 \times 10^{5}$ & $7.7 \times 10^{4}$ & $4.8 \times 10^{5}$ & $1.1 \times 10^{5}$ & $4.6 \times 10^{4}$ & $2.2 \times 10^{3}$ & $2.1 \times 10^{6}$ & $6.4 \times 10^{5}$ \\
\hline C1 Fluoranthenes/Pyrene & 18 & 23 & $5.6 \times 10^{5}$ & $1.0 \times 10^{5}$ & $1.3 \times 10^{6}$ & $1.9 \times 10^{5}$ & $1.9 \times 10^{5}$ & $2.0 \times 10^{4}$ & $5.5 \times 10^{6}$ & $9.1 \times 10^{5}$ \\
\hline C2 Fluoranthenes/Pyrene (P) & 16 & 19 & $6.5 \times 10^{5}$ & $1.5 \times 10^{5}$ & $1.4 \times 10^{6}$ & $2.0 \times 10^{5}$ & $2.0 \times 10^{5}$ & $2.5 \times 10^{4}$ & $5.3 \times 10^{6}$ & $8.8 \times 10^{5}$ \\
\hline C3 Fluoranthenes/Pyrene (P) & 18 & 20 & $7.7 \times 10^{5}$ & $1.8 \times 10^{5}$ & $1.4 \times 10^{6}$ & $2.1 \times 10^{5}$ & $2.3 \times 10^{5}$ & $2.8 \times 10^{4}$ & $4.7 \times 10^{6}$ & $9.6 \times 10^{5}$ \\
\hline C4 Fluoranthenes/Pyrene (P) & 17 & 19 & $8.2 \times 10^{5}$ & $2.3 \times 10^{5}$ & $1.9 \times 10^{6}$ & $2.6 \times 10^{5}$ & $2.3 \times 10^{5}$ & $3.0 \times 10^{4}$ & $8.4 \times 10^{6}$ & $9.8 \times 10^{5}$ \\
\hline C1 Benz(a)anthracenes/Chrysene/Triphenylenes (P) & 17 & 19 & $8.0 \times 10^{5}$ & $1.8 \times 10^{5}$ & $2.1 \times 10^{6}$ & $3.0 \times 10^{5}$ & $2.4 \times 10^{5}$ & $2.3 \times 10^{4}$ & $1.0 \times 10^{7}$ & $1.4 \times 10^{6}$ \\
\hline C2 Benz(a)anthracenes/Chrysene/Triphenylenes (P) & 18 & 21 & $1.0 \times 10^{6}$ & $1.7 \times 10^{5}$ & $1.9 \times 10^{6}$ & $3.0 \times 10^{5}$ & $2.1 \times 10^{5}$ & $2.3 \times 10^{4}$ & $8.7 \times 10^{6}$ & $1.9 \times 10^{6}$ \\
\hline C3 Benz(a)anthracenes/Chrysene/Triphenylenes (P) & 18 & 20 & $9.7 \times 10^{5}$ & $2.3 \times 10^{5}$ & $1.7 \times 10^{6}$ & $2.2 \times 10^{5}$ & $2.3 \times 10^{5}$ & $2.7 \times 10^{4}$ & $6.2 \times 10^{6}$ & $8.9 \times 10^{5}$ \\
\hline C4 Benz(a)anthracenes/Chrysene/Triphenylenes (P) & 19 & 22 & $8.3 \times 10^{5}$ & $2.3 \times 10^{5}$ & $1.5 \times 10^{6}$ & $3.6 \times 10^{5}$ & $2.1 \times 10^{5}$ & $3.3 \times 10^{4}$ & $5.4 \times 10^{6}$ & $2.7 \times 10^{6}$ \\
\hline Dibenzothiophene $(G)$ & 15 & 28 & $2.2 \times 10^{5}$ & $1.7 \times 10^{4}$ & $4.2 \times 10^{5}$ & $4.7 \times 10^{4}$ & $1.5 \times 10^{4}$ & $9.7 \times 10^{2}$ & $3.1 \times 10^{6}$ & $4.2 \times 10^{5}$ \\
\hline C1 Dibenzothiophenes (G) & 15 & 28 & $2.2 \times 10^{5}$ & $2.0 \times 10^{4}$ & $4.3 \times 10^{5}$ & $5.0 \times 10^{4}$ & $2.1 \times 10^{4}$ & $2.1 \times 10^{3}$ & $2.1 \times 10^{6}$ & $3.9 \times 10^{5}$ \\
\hline C2 Dibenzothiophenes (G) & 15 & 28 & $3.0 \times 10^{5}$ & $3.2 \times 10^{4}$ & $1.1 \times 10^{6}$ & $7.9 \times 10^{4}$ & $4.0 \times 10^{4}$ & $1.0 \times 10^{4}$ & $1.1 \times 10^{7}$ & $6.2 \times 10^{5}$ \\
\hline C3 Dibenzothiophenes & 14 & 27 & $2.9 \times 10^{5}$ & $6.6 \times 10^{4}$ & $4.1 \times 10^{5}$ & $1.3 \times 10^{5}$ & $7.8 \times 10^{4}$ & $1.6 \times 10^{4}$ & $1.4 \times 10^{6}$ & $1.0 \times 10^{6}$ \\
\hline C4 Dibenzothiophenes & 14 & 26 & $2.9 \times 10^{5}$ & $9.7 \times 10^{4}$ & $4.3 \times 10^{5}$ & $1.9 \times 10^{5}$ & $9.4 \times 10^{4}$ & $2.6 \times 10^{4}$ & $2.1 \times 10^{6}$ & $1.9 \times 10^{6}$ \\
\hline Overall & & & $3.9 \times 10^{5}$ & $8.3 \times 10^{4}$ & $4.6 \times 10^{5}$ & $1.1 \times 10^{5}$ & & & & \\
\hline
\end{tabular}

